\title{
Title: Novel indole-based tambjamine-analogues induce apoptotic lung cancer cell death through p38 mitogen-activated protein kinase activation
}

\author{
Authors and Affiliations \\ Pilar Manuel-Manresa ${ }^{1, \neq}$, Luís Korrodi-Gregório ${ }^{1,2, \neq}$, Elsa Hernando ${ }^{3}$, Alberto Villanueva ${ }^{4}$, David \\ Martínez-García ${ }^{1}$, Ananda M. Rodilla ${ }^{1}$, Ricard Ramos ${ }^{5}$, Margarida Fardilha ${ }^{2}$, Juan Moya ${ }^{5}$, Roberto \\ Quesada $^{3}$, Vanessa Soto-Cerrato ${ }^{1,}$, Ricardo Pérez-Tomás ${ }^{1, *}$ \\ ${ }^{1}$ Cancer Cell Biology Research Group, Department of Pathology and Experimental Therapeutics, Faculty of \\ Medicine, University of Barcelona, Barcelona, Spain \\ ${ }^{2}$ Laboratory of Signal Transduction, Department of Medical Sciences, Institute for Research in Biomedicine - \\ iBiMED, Health Sciences Program, University of Aveiro, Aveiro, Portugal \\ ${ }^{3}$ Department of Chemistry, University of Burgos, Burgos, Spain \\ ${ }^{4}$ Program Against Cancer Therapeutic Resistance (ProCURE), Catalan Institut of Oncology ICO, Bellvitge \\ Biomedical Research Institute IDIBELL, L'Hospitalet \\ ${ }^{5}$ Department of Thoracic Surgery and University of Barcelona, Hospital Universitari de Bellvitge, L'Hospitalet \\ de Llobregat, Barcelona, Spain \\ ${ }^{\ddagger}$ Authors have contributed equally to the work
}

Running title: Cancer cell death induced by small molecule anionophores

Keywords: lung cancer, tambjamine analogues, anionophores, anion transporter, apoptosis

Abbreviations: BCA, bicinchoninic acid; Bcl2, B-cell lymphoma 2; BSA, bovine serum albumin; CDDP, cis-diamminedichloroplatinum (II); CSC, cancer stem cells; $C_{T}$, threshold cycle; DHE, dihydroethidium; DK-SFM, defined keratinocyte-serum free medium; DMSO, dimethyl sulfoxide; EGFR, epidermal growth factor receptor; FBS, fetal bovine serum; IACUC, Institutional Animal Care and Use Committee; IAPS, inhibitor of apoptosis proteins; IC, inhibitory concentration; PC, primary cultures; LC, lung cancer; MAPK, mitogen activated protein kinase; MOMP, mitochondrial outer membrane permeabilization; MTT, methylthiazoletetrazolium; PARP, poly ADP ribose polymerase; PVDF, polyvinylidene difluoride; ROS, reactive oxygen species; SD, standard deviation; SDS, sodium dodecyl sulfate; TBHP, tert-Butyl hydroperoxide.

Financial Support: This work was supported by grants from the Spanish Government and EU funds through the Fondo de Investigaciones Sanitarias (FIS, Project PI13/00089) and from La Marató de TV3 Foundation (Project 20132730) to R. Pérez-Tomás. R. Ramos was supported by the Sociedad Española de Neumología y Cirugía Torácica (SEPAR, Project 017/2013), R. Quesada by the Consejería de Educación de la Junta de Castilla y León (Project BU340U13) and by the La Marató de TV3 Foundation (Project 20132732) and A. Villanueva by the FIS (Project PI13-01339). This work was also supported by an individual grant from FCT (Fundação para a Ciência e a Tecnologia) to L. KorrodiGregório (SFRH/BPD/91766/2012).

*Corresponding authors: Cancer Cell Biology Research Group, Department of Pathology and Experimental Therapeutics, Faculty of Medicine, University of Barcelona, C./ Feixa Llarga s/n 08907 Hospitalet de Llobregat. Barcelona, Spain. Correspondence should be addressed to rperez@ub.edu (Ricardo Pérez-Tomás) or vsoto@ub.edu (Vanessa Soto-Cerrato).

Conflicts of Interest: The authors declare no conflict of interest.

Category: Small Molecule Therapeutics

Word Count: 5528 words; Number of Figures/Tables: 6

Supplementary Figures/Tables: 44 


\section{Abstract}

Lung cancer has become the leading killer cancer worldwide, due to late diagnosis and lack of efficient anticancer drugs. We have recently described novel natural-derived tambjamine analogues that are potent anion transporters capable of disrupting cellular ion balance, inducing acidification of the cytosol and hyperpolarization of cellular plasma membranes. Although these tambjamine analogues were able to compromise cell survival, their molecular mechanism of action remains largely unknown. Herein we characterize the molecular cell responses induced by highly active indole-based tambjamine analogues treatment in lung cancer cells. Expression changes produced after compounds treatment comprised genes related to apoptosis, cell cycle, growth factors and its receptors, protein kinases and topoisomerases, among others. Dysregulation of BCL2 and BIRC5/survivin genes suggested the apoptotic pathway as the induced molecular cell death mechanism. In fact, activation of several pro-apoptotic markers (caspase 9, caspase 3 and PARP) and reversion of the cytotoxic effect upon treatment with an apoptosis inhibitor (Z-VAD-FMK) were observed. Moreover, members of the $\mathrm{Bcl}-2$ protein family suffered changes after tambjamine analogues treatment, with a concomitant protein decrease towards the pro-survival members. Besides this, it was observed cellular accumulation of ROS upon compound treatment and an activation of the stress-kinase p38 MAPK route that, when inhibited, reverted the cytotoxic effect of the tambjamine analogues. Finally, a significant therapeutic effect of these compounds was observed in subcutaneous and orthotopic lung cancer mice models. Taken together, these results shed light on the mechanism of action of novel cytotoxic anionophores and demonstrate the therapeutic effects against lung cancer. 


\section{Introduction}

Lung cancer (LC) is the most common cancer that threatens human health, accounting for more than 19\% of all cancer deaths in 2012 (1). Health systems have standardized clinical procedures for treating LC patients being surgery, conventional platinum-based doublet chemotherapy (e.g. carboplatin/taxol or pemetrexed) and targeted therapies (e.g. erlotinib and other novel anti-EGFR mutated receptors) the main options (2). Nevertheless, conventional chemotherapy shows low efficacy with important treatment-related toxicity. Therefore, development of novel efficient drugs is urgently needed. In this regard, the diversity of marine environment provides an extraordinary array of biologically active metabolites for the development of new anticancer therapeutics. In late 2007, trabectedin (Yondelis, PharmaMar) became the first marine anticancer drug to be approved in the European Union (3). Over the past decade, there has been an increase in the number of new anticancer lead compounds from marine life that have entered in human clinical trials (4). Nowadays, more than 592 marine compounds are included in the pipeline of modern pharmaceuticals discovery programs, showing promising antitumor and cytotoxic activities (5). The natural alkaloids tambjamines were originally isolated from marine invertebrates including bryozoans, nudibranchs and ascidians (6). Tambjamines possess a wide spectrum of pharmacological properties and seem to be involved in the chemical defense mechanisms of the organisms from which they derive (6). Structurally, these alkaloids are characterized by a 4-methoxybipyrrole moiety shared by other families of natural products such as the prodiginines (7). It is already well known that prodiginines have pro-apoptotic activity against several cancer cells including lung, breast and hematopoietic, showing the ability to overcome the multidrug resistance phenotype (8-10). Tambjamines, like prodiginines, are very efficient anion exchangers (anionophores) in model liposomes promoting both chloride and bicarbonate transport, which are the most abundant anions 
in biological environments $(7,11,12)$. Their anionophoric activity has an impact on cellular ion homeostasis, intracellular pH levels and cell survival $(11,12)$. Recently, we have demonstrated that synthetic tambjamine analogues induce intracellular $\mathrm{pH}$ acidification and cytotoxicity in LC cells and human-derived cancer stem cells (CSCs), and how this is related to their anion transport abilities (13). Cancer stem cells are defined as immortal cells within a tumor that have the ability to perpetuate themselves through self-renewal, to spawn differentiated progeny (non-CSCs) and contribute to acquired chemotherapy resistance in cancer (14). Moreover, their facilitated transport activity triggers hyperpolarization of plasma membrane in LC cells and differentiation and cell death in CSCs, leading to an effective elimination of this tumor subpopulation (13).

Although substantial work has already been done concerning chemical characterization of tambjamines and the impact of their anionophoric properties in cell survival and differentiation, their molecular mechanism of action still remains unclear. Here, we report a detailed study of how cancer cells respond to several highly active indole-based tambjamine analogues at the transcript and protein levels. Moreover, we have elucidated the molecular cell death mechanism induced by these compounds as well as assessed their therapeutic effect in vivo in several LC mouse models. 


\section{Materials and Methods}

Compounds synthesis. Compounds 1-11 were synthesized by condensation of , 5-(1H-indole-2-yl)-3methoxy-1H-pyrrole-2-carbaldehyde with the appropriate amine using acetic acid as catalyst. Detailed synthetic procedures and characterization data are provided in the supplementary information (S1-S36).

Cell lines and culture conditions. The human LC cell lines, adenocarcinoma (A549, CCL185 ${ }^{\mathrm{TM}}$ ), squamous carcinoma (SW900, HTB59 ${ }^{\mathrm{TM}}$ ), small cell carcinoma (DMS53, CRL2062 ${ }^{\mathrm{TM}}$ ) and large cell carcinoma (H460, HTB177 ${ }^{\mathrm{TM}}$ ) were obtained from American Tissue Culture Collection (ATCC, Manassas, VA, USA). All cell lines were tested and authenticated by ATCC using short tandem repeat analysis. All cell lines were cultured (passage number 10-25) following ATCC recommended media (Biological Industries, Beit Haemek, Israel) supplemented with 10\% heat-inactivated fetal bovine serum (FBS; Gibco, Thermo Fisher Scientific Inc. Waltham, MA, USA), $100 \mathrm{U} / \mathrm{ml}$ penicillin, 100 $\mathrm{g} / \mathrm{ml}$ streptomycin, and $2 \mathrm{mM} \mathrm{L-glutamine,} \mathrm{all} \mathrm{from} \mathrm{Biological} \mathrm{Industries.} \mathrm{Cells} \mathrm{were} \mathrm{grown} \mathrm{at} 37^{\circ} \mathrm{C}$ in a humidified incubator (Thermo Fisher Scientific Inc.) with 5\% $\mathrm{CO}_{2}$ atmosphere and were mycoplasmafree.

Establishment of LC patient-derived primary cultures. This protocol was approved by the local Ethics Committee (PR003/13) and, upon signed informed consent; fresh human LC tissues were obtained from 20 patients with primary LC (age range 55-81 years) undergoing lobectomy or pneumonectomy at Bellvitge University Hospital from April 2013 to March 2014. Histological diagnosis was determined based on microscopic features of carcinoma cells. Freshly obtained tumor tissue (within 1-2 hours after surgical removal) was washed in RPMI-1640 medium (Biological 
Industries) containing $100 \mathrm{U} / \mathrm{ml}$ penicillin and $100 \mu \mathrm{g} / \mathrm{ml}$ streptomycin. Blood vessels and connective tissue were carefully removed and the cancerous area was then minced into small pieces less than 1 $\mathrm{mm}^{3}$ using a scalpel. Chopped tissue was resuspended in RPMI-1640 medium containing collagenase II (Sigma-Aldrich, Merck KGaA, Darmstadt, Germany) at a concentration of $200 \mathrm{U} / \mathrm{ml}$ and digested for 2-4 hours at $37^{\circ} \mathrm{C}$ in a humidified incubator. The enzymatic digestion was stopped when most of the cells were in a single cell suspension. Following two washes in RPMI-1640, cells were transferred into standard tissue culture coated flasks (TPP, Trasadingen, Switzerland) and cultured in the Defined Keratinocyte-Serum Free Medium (DK-SFM, Gibco, Thermo Fisher Scientific Inc.) supplemented with $100 \mathrm{U} / \mathrm{ml}$ penicillin, $100 \mathrm{\mu g} / \mathrm{ml}$ streptomycin, $2 \mathrm{mM} \mathrm{L-glutamine,} 20$ ng/ml EGF (Sigma-Aldrich, Merck KGaA), $10 \mathrm{ng} / \mathrm{ml}$ basic-FGF (Sigma-Aldrich, Merck KGaA), 2\% B27 (Gibco, Thermo Fisher Scientific Inc.) and $0.25 \mathrm{mg} / \mathrm{ml}$ amphotericin B (Sigma-Aldrich, Merck KGaA). All LC patient-derived cultures were maintained at $37^{\circ} \mathrm{C}$ in a humidified incubator (Thermo Fisher Scientific Inc.) with $5 \% \mathrm{CO}_{2}$. Culture medium was changed every 2-3 days. Cells were passaged after detachment with TrypLE $^{\mathrm{TM}}$ Express (Invitrogen, Thermo Fisher Scientific Inc.), when the cells reached 80-90\% confluence. We were able to establish two LC primary cultures (PC), one derived from an adenocarcinoma tumor (PC\#8) and the other derived from a squamous cell carcinoma (PC\#13), which were used for subsequent viability assays. LC patient-derived PC were characterized by immunofluorescence, according to their epithelial or mesenchymal biomarker expression, using cytokeratin-8 (Cat\#IF13, Oncogene, Merck KGaA) for the epithelial phenotype and vimentin (Cat\#3932, Cell Signaling Technology Inc., Danvers, MA, USA) for the mesenchymal phenotype.

Cell viability assays. Cell viability was evaluated using the methylthiazoletetrazolium (MTT, SigmaAldrich, Merck KGaA) colorimetric assay. Cells were harvested $\left(10^{4}\right.$ cells/well) in 96 -well plates in a final volume of $100 \mu \mathrm{l}$ and allowed to grow overnight. At the following day, vehicle solution (DMSO, 
Sigma-Aldrich, Merck KGaA) or experimental compounds were added at a single point (10 $\mu \mathrm{M})$ or at different ranging concentrations $(0.8-100 \mu \mathrm{M}$ for compounds 1 and 2, 1.6-200 $\mu \mathrm{M}$ for cisplatin) to the assay plate. Cells were incubated for 24 hours and after the treatment period, $10 \mu \mathrm{l}$ of MTT (5 $\mathrm{mg} / \mathrm{ml}$ ) were added and the plates were incubated for 4 hours at $37^{\circ} \mathrm{C}$. Crystals were dissolved in $100 \mu \mathrm{l}$ of DMSO, after which the reading was taken spectrophotometrically at $570 \mathrm{~nm}$ using a multiwell plate reader (Multiskan FC, Thermo Fisher Scientific Inc.). Cell viability and inhibitory concentration (IC) values were obtained using GraphPad Prism V5.0 for Windows (Graphpad Software, San Diego, CA, USA). All data are shown as the mean value \pm standard deviation (S.D.) of three independent experiments. Statistical analysis (one-way ANOVA) was carried out with the Statgraphics plus 5.1. Statistical package (Manugistics, Rockville, MD, USA). Cisplatin was bought from Alfa Aesar (Thermo Fisher Scientific Inc.).

Gene expression analysis. The A549 cell line was seeded at a density of $2 \times 10^{5}$ cells in $60 \mathrm{~mm}$ plates and allowed to grow for 24 hours. Subsequently, cells were exposed to the $\mathrm{IC}_{50}$ of the tambjamine analogues 1 or 2 for 6 or 16 hours. Total RNA was extracted and purified using the column-based RNeasy Mini Kit (QIAGEN, Hilden, Germany), according to the manufacture's protocol. Total RNA concentration and purity was checked in a nano spectrophotometer (Implen GmbH, München, Germany) and integrity was analyzed using an Agilent 2100 Bioanalyzer (Agilent Technologies, Santa Clara, CA, USA). For the reverse transcription, $1 \mu \mathrm{g}$ of total RNA was used for CDNA synthesis using a mixture of random hexamers and oligo-dT primers and following the $\mathrm{RT}^{2}$ First Strand Kit protocol (QIAGEN). The Human RT² RNA Quality Control PCR Array (PAHS-999ZA format A, QIAGEN) was used to assess the cDNA quality and to check for genomic DNA contamination in an ABI PRISM 7700 Sequence Detection System (Applied Biosystems, Thermo Fisher Scientific Inc.). For gene expression analysis, cDNAs of control and treated cells were mixed with $\mathrm{RT}^{2}$ SYBR ROX mastermix (QIAGEN) and 
dispensed in the $\mathrm{RT}^{2}$ Profiler PCR Array of Human Cancer Drug Targets (PAHS-507A format E, QIAGEN). After 2 minutes centrifugation at $300 \mathrm{~g}$, the array was placed in an ABI PRISM 7900HT realtime PCR system (Applied Biosystems, Thermo Fisher Scientific Inc.) and the analysis was carried out according to manufacture's instructions. Dissociation curves (melting curves) were carefully analyzed using SDS software v2.3 and RQ Manager v1.2 (both from Applied Biosystems, Thermo Fisher Scientific Inc.) in order to choose only the highly specific reaction products in the downstream analysis. The threshold cycle $\left(C_{T}\right)$ values obtained were analyzed in QIAGEN Data Analysis Center (QIAGEN) to retrieve the fold-regulation values for each gene. The network of most altered genes upon compounds treatment was produced in Cytoscape open-source software (Cytoscape Consortium, San Diego, CA, USA) (15) with data extracted from Gene Network Central (GNC-Pro, QIAGEN).

Western blot analysis. A549 cells were seeded in $100 \mathrm{~mm}$ culture plates ( $10^{6}$ cells) and allowed to grow for 24 hours. Afterwards, they were exposed to compounds $1,2\left(I C_{25}, I C_{50}\right.$ and $I C_{75}$ values) or 3 $(10 \mu \mathrm{M})$ for 24 hours. Total protein extracts were obtained from cells by the addition of lysis buffer containing $0.1 \%$ SDS, $1 \%$ NP-40, $0.5 \%$ sodium deoxycholate, $50 \mathrm{mM}$ sodium fluoride, $40 \mathrm{mM} \beta$ glycerophosphate, $200 \mu \mathrm{M}$ sodium orthovanadate, $1 \mathrm{mM}$ phenylmethylsulfonyl fluoride and protease inhibitor cocktail (Roche Diagnostics, Basel, Switzerland) in 1X PBS. Protein concentration was determined by BCA protein assay (Pierce, Thermo Fisher Scientific Inc.) using bovine serum albumin (BSA, Pierce, Thermo Fisher Scientific Inc.) as a standard. For Western blot analysis, $30 \mu \mathrm{g}$ of protein extracts were first separated by SDS-polyacrylamide gel electrophoresis and transferred to Immobilon-P polyvinylidene difluoride (PVDF) membranes (EMD Millipore, Merck KGaG). Membranes were blocked in either $5 \%$ dry milk or BSA, both diluted in TBS-Tween $(50 \mathrm{mM}$ Tris- $\mathrm{HCl}$ $\mathrm{pH}$ 7.5, $150 \mathrm{mM} \mathrm{NaCl}, 0.1 \%$ Tween-20) for 1 hour and then incubated overnight with primary 
antibodies, according to the manufacturer's instructions. Antibodies were obtained from the following sources: anti-Caspase-3 (Cat\#9662), anti-cleaved-Caspase-3 (Cat\#9661), anti-Caspase-9 (Cat\#9502), anti-PARP1 (Cat\#9542), anti-Bak (Cat\#6947), anti-Bax (Cat\#2772), anti-Bcl-2 (Cat\#4223), anti-Mcl-1 (Cat\#4572), anti-phospho-p38 MAPK (Thr180/Tyr182, Cat\#4511), anti-p38 MAPK (Cat\#9212) and anti-Survivin (Cat\#2808), were all obtained from Cell Signaling Technology Inc.; antiActin (I-19, Cat\#sc-1616) from Santa Cruz Biotechnology Inc. (Santa Cruz, CA, USA) and anti-Vinculin (Cat\# V-4505) from Sigma-Aldrich, Merck KGaA. Antibody binding was detected with goat antimouse IgG-HRP (Cat\#sc-2005), goat anti-rabbit IgG-HRP (Cat\#sc-2004) or donkey anti-goat IgG-HRP (Cat\#sc-2020) all from Santa Cruz Biotechnology Inc. and the ECL detection kit (Amersham, GE Healthcare, Little Chalfont, UK). Actin or vinculin were used as gel loading controls. The results shown are representative of Western blot data obtained from at least three independent experiments. Images were captured on an Image Quant LAS 500 (GE Healthcare) and band densitometries were retrieved using the Image Studio Lite software (v5.0, Li-COR Biosciences, Lincoln, NE, USA).

Inhibitor assays. Determination of viable cells (A549 cell line) after different inhibitors treatment was performed using the dual DNA intercalating fluorescent dyes kit MUSE ${ }^{\text {TM }}$ Cell Count \& Viability Assay (EMD Millipore, Merck KGaG). In brief, $2 \times 10^{5}$ cells/well in DMEM medium were seeded in a 6 wellplate. After 24 hours, tambjamine analogues 1 and 2 at higher $I C_{75}$ value were added, and incubated for 6 hours in a 5\% $\mathrm{CO}_{2}$-cell culture incubator (Thermo Fisher Scientific Inc.). A pan-caspases inhibitor (Z-VAD-FMK; BD Bioscience, Franklin Lakes, NJ, USA) or a p38 MAPK inhibitor (SB202190; Cell Signaling Technology Inc.) was added at 20 and $30 \mu \mathrm{M}$ respectively, 2 hours before compound 1 or 2 exposure. Afterwards, cells were collected and were incubated with the Cell Count \& Viability reagent for 5 minutes. Viability of treated cells was analyzed using the flow cytometry based MUSE ${ }^{\mathrm{TM}}$ 
Cell Analyzer (EMD Millipore, Merck KGaA) according to the manufacture's protocol. Viability was calculated as a percentage related to control cells and results shown were obtained from at least three independent experiments. Statistical analysis (one-way ANOVA) was carried out with the Statgraphics plus 5.1. Statistical package (Manugistics).

Evaluation of reactive oxygen species (ROS) formation. Quantitative measurements of ROS in A549 cells were performed using the MUSE ${ }^{\mathrm{TM}}$ Oxidative Stress Kit (EMD Millipore, Merck KGaG), based on dihydroethidium (DHE), a well characterized reagent that has extensively been used to detect reactive oxidative species in cellular populations. In brief, $2 \times 10^{5}$ cells/well in DMEM medium were seeded in a 6 well-plate. 24 hours after seeding, tambjamine analogues 1 and 2 at higher $I C_{75}$ value were added, and incubated for 6 hours in a 5\% $\mathrm{CO}_{2}$-cell culture incubator (Thermo Fisher Scientific Inc.). A positive control, named tert-Butyl hydroperoxide (TBHP, Sigma-Aldrich, Merck KGaA) was used at $2.5 \mathrm{mM}$ for 2 hours, and the non-transporter tambjamine analogue (compound 3) was used as a negative control at $10 \mu \mathrm{M}$ for 6 hours. Afterwards, cells were harvested and processed with the Oxidative Stress kit according to the manufacture's protocol. The percentage of ROS positive and negative cells was calculated and results shown were obtained from at least three independent experiments. Statistical analysis (one-way ANOVA) was carried out with the Statgraphics plus 5.1. Statistical package (Manugistics).

In vivo evaluation of indole-based tambjamine-analogues therapeutic effect. For the purpose of this article, two murine models have been designed: the subcutaneous and the orthotopic model systems. 5-weeks-old female Crl:NU-Foxn1nu mice strain (Envigo, Huntingdon, UK) were used in this study. All animal studies were performed in accordance with the guidelines approved by the Institutional Animal Care and Use Committee (IACUC). To generate the subcutaneous xenograft 
model, mice were subcutaneously implanted with $4.5 \times 10^{6}$ DMS53 cells suspended in a 1:1 solution of RPMI-1460:Matrigel (BD Bioscience). For the orthotopic model, subcutaneous xenografts of DMS53 in exponential growth from three different animals were aseptically isolated and placed at room temperature in DMEM supplemented with $10 \%$ FBS plus $50 \mathrm{U} / \mathrm{ml}$ penicillin and $50 \mathrm{mg} / \mathrm{ml}$ streptomycin and the surgical resection tumors were implanted in Crl:NU-Foxn1nu mice following previously reported procedures $(16,17)$. Briefly, mice were anesthetized with a continuous flow of $1 \%$ to $3 \%$ isoflurane/oxygen mixture $(2 \mathrm{~L} /$ minutes) and subjected to right thoracotomy. Mice were situated in left lateral decubitus position and a small transverse skin incision (around 5 to $8 \mathrm{~mm}$ ) was made in the right chest-wall. Chest muscles were separated by a sharp dissection and costal and intercostal muscles were exposed. An intercostal incision of 2-4 $\mathrm{mm}$ on the third or fourth rib on the chest-wall was made and a small tumor piece of $2-4 \mathrm{~mm}^{3}$ was introduced into the chest cavity. The tumor specimen was deposited between the second and the third lung lobule. Next, the chest-wall incision was closed with surgery staples, and finally chest muscles and skin were closed. Mice were inspected twice a week, and monitored for the presence of breathing problems.

Mice bearing homogeneous subcutaneous tumors (approximately 150-200 $\mathrm{mm}^{3}$ ) were randomly allocated to three treatment groups ( $n=7 /$ treatment). For DMS53 orthotopic derived model, mice were randomly allocated ( $n=6 /$ treatment) and the treatment started 30 days after tumor implantation. Compounds 1 and 2 were diluted in 7.5\% DMSO/0.8\% Tween-80. All treatments were intraperitoneally administrated at a dose of $6 \mathrm{mg} / \mathrm{kg}$ in alternating days during 20 days. Tumor growth was recorded 2 to 3 times per week starting from the first day of treatment (day 0) and tumor volume (in $\mathrm{mm}^{3}$ ), estimated according to the formula $\vee 1 / 4(a b 2) / 2$, (a: length or biggest diameter; b: width or smallest diameter). After the final dose the treatment, animals were sacrificed and tumors were dissected out and weighed. 
Liver and kidney samples were fixed in $4 \%$ paraformaldehyde overnight, embedded in paraffin, and cut into sections $(5 \mu \mathrm{m})$. Paraffin-embedded sections were deparaffinized in xylene and gradually rehydrated for hematoxylin and eosin staining. Stained liver and kidney sections were gradually dehydrated and mounted for hepatotoxicity and nephrotoxicity examination, respectively.

\section{Results}

Indole-based tambjamine analogues are cytotoxic against LC cells.

To measure the inhibitory effect of novel tambjamine analogues (1-11; supplementary Fig. S1) on tumor cell proliferation, we have first performed a single-point MTT assay at $10 \mu \mathrm{M}$ in four commonly used LC cell lines (A549, DMS53, SW900 and H460). After 24 hours of treatment, most of the compounds showed significant cell viability decrease, being compounds 1 and 2 the most promising anticancer agents (Fig. $1 \mathrm{~A})$. Then, half-maximal inhibitory concentration $\left(\mathrm{IC}_{50}\right)$ values of the selected compounds were calculated in the same cell lines as well as in both adenocarcinoma and squamous patient-derived PC (Fig. 1B and Supplementary Fig. S37 and S38). The two selected compounds had similar potency against all the cell lines in the study, showing $I C_{50}$ values below $10 \mu \mathrm{M}$. When compared with cis-diamminedichloroplatinum (II) (CDDP), commonly known as cisplatin, the standard chemotherapeutic agent used for LC treatment in the clinic, the $\mathrm{IC}_{50}$ values of this compound were all much higher than the ones of compounds 1 and 2 . Interestingly, LC patientderived PC were more sensitive to our compounds, showing $I C_{50}$ values below $5 \mu \mathrm{M}$ while for cisplatin they were higher than $45 \mu \mathrm{M}$ (Fig. 1B). This $I_{50}$ value for cisplatin is in accordance with published data $(18,19)$. Therefore, the selected tambjamine analogues displayed better cytotoxic activity against a panel of LC cell lines and PC than the current clinical standard treatment.

Indole-based tambjamine analogues alter the expression of several cellular key genes. 
To identify gene expression changes at the cellular level we have used a profiler array consisting of 84 genes that are known cancer drug targets. The selected compounds 1 and 2 were tested in the adenocarcinoma cell line $A 549$ at their respective $I C_{50}$ values during two incubation periods (6 and 16 hours). Both compounds have produced changes in several genes from diverse functional groups (Table 1). In general, the observed alterations (higher than 1.5 fold-regulation in at least 2 conditions) were more evident at 16 hours with a clear tendency to genes down-regulation. In total 26 genes were modulated, in which 20 were down-regulated by compounds 1 or 2 exposure. Downregulated genes included those related to: apoptosis, cell cycle, growth factors and receptors, protein kinases, topoisomerases, to name a few. Especially relevant for cell viability were genes related to cell death like the anti-apoptotic $B L C 2$ (B-cell lymphoma 2) and the inhibitor of apoptosis (IAP) family member BIRC5/Survivin, as well as those related to cell cycle (e.g. CDK1 and CDK2). The remaining 6 genes were up-regulated and related to drug metabolism, protein kinases, transcription factors, cathepsins and heat shock proteins (Table 1). Concerning genes that are altered more than 3 fold-regulation in at least 2 conditions, we have obtained CDC25A, FIGF/VEGFD, IGF1, NFKB1 and PTGS2/Cox-2. Six randomly selected genes were re-confirmed by another methodology (Supplementary Table S1 and Fig. S39). Similar results were obtained using another LC cell line from squamous carcinoma SW900 (77\% gene match, Supplementary Table S2), suggesting that they may be implicated in tambjamine mechanism of action. Interestingly, three genes that were previously altered more than 3-fold in A549 cell line (FIGF, IGF and NFKB1) were not altered, IGF and NFKB1 or inversely regulated, FIGF, in the SW900 cell line. We further analyzed the altered genes using protein-protein interaction data and functional data extracted from Gene Network Central (GNC-Pro, QIAGEN). The results showed that compounds-modulated genes formed a highly connected network (Fig. 2), suggesting that these genes work as a functional module at the molecular level. The network also showed that NFKB1 and TP53 are hubs (densely connected nodes) which integrate signals from 
the other nodes. Although these genes are hubs in our system, we did not observe any consistent transcriptional change in both cell line models.

Indole-based tambjamine analogues induce apoptosis in lung cancer cells.

Since we have obtained alterations in genes related to apoptosis and cell cycle and these processes are deeply involved in cell viability we checked if these mechanisms are activated in our cellular model. We were unable to detect any cell cycle arrest for either compounds tested, whereas cisplatin produced the well-documented G2/M phase arrest (Supplementary Fig. S40) (20). To elucidate if apoptosis is the molecular mechanism of cell death induced by these compounds, the expression levels of apoptosis-related proteins were analyzed by Western blot, since a decrease in the anti-apoptotic proteins $\mathrm{Bcl}-2$ and survivin was detected in the gene expression array. The adenocarcinoma cell line $A 549$ was treated with compounds 1 or 2 at the $I C_{25}, I_{50}$ and $I C_{75}$ concentrations for 24 hours. The results showed that, at higher concentrations, compounds activated the proteolysis of both initiator pro-caspase-9 (CASP9 gene) and executioner pro-caspases3 (CASP3 gene) (Fig. 3a). Moreover, activation of the final apoptotic executioner PARP was also induced. Similar results were also obtained in the small cell carcinoma cell line DMS53 (Supplementary Fig. S41). In addition, we checked the levels of the apoptotic complexes Bak/Mcl-1 (BAK/MCL1 genes) and Bax/BCl-2 (BAX/BCL2 genes). The pro-apoptotic effectors of the mitochondrial pathway (Bak and Bax) showed an increase in their protein levels after 24 hours treatment. On the contrary, $\mathrm{Mcl}-1$ and $\mathrm{Bcl}-2$ that are pro-survival members of the $\mathrm{Bcl}-2$ family of proteins, presented a decrease on their protein levels after treatment (Fig. 3a-3c). The results for Bcl-2 protein expression corroborated the gene expression decrease previously observed in the profiler array (Table 1). Also in accordance with those results, down-regulation of the key oncogene BIRC5/Survivin was observed at the protein level by Western blot. A549 cells treated with compounds 1 or $2\left(I C_{25}, I C_{50}\right.$ and IC 75$)$ 
during 24 hours showed a dose-dependent decrease of survivin, indicating that this protein might play an important role on the cell death fate after compounds exposure (Fig. 3a).

To further investigate the involvement of caspases activation on compounds-mediated apoptosis, A549 cells were treated 2 hours with a broad-spectrum caspase inhibitor, Z-VAD-FMK, and then with compounds 1 or 2 for 6 hours. Cell viability was then assessed by flow cytometry and results showed that the decrease in cell viability induced by compounds treatment was significantly reversed by the pan-caspase inhibitor Z-VAD-FMK (Fig. 3d), corroborating that apoptosis is involved in compoundsinduced cell death.

\section{Cytotoxic effect of compounds is triggered via a ROS-activated stress kinase pathway.}

The unbalance of ionic homeostasis produced by tambjamine analogues (13), could be initiating ROS production. Therefore, we tested whether compounds 1 and 2 could promote ROS accumulation in cells. Interestingly, after 6 hours treatment with compounds 1 or 2 (at higher $I_{75}$ value) there is a significant ROS formation compared with the non-treated condition or when the non-transporter tambjamine analogue (compound 3 ) is used (Fig. 4a). The effect of compounds is similar to the commonly used ROS stressor TBHP. The p38 MAPK signaling is one of the stress sensor pathways downstream of ROS formation that plays an essential role in inflammation, cell differentiation, growth and death. Thus, we have investigated by Western blot the phosphorylation of p38 MAP kinase after treatment with indole-based tambjamine analogues 1, 2 or 3 in A549 cells. After 24 hours treatment with compounds 1 or $2\left(I C_{25}, I C_{50}\right.$ and $\left.I C_{75}\right)$, p38 MAPK (MAPK14 gene) phosphorylation increased in a dose response manner without any alteration in the total protein expression levels (Fig. 4b). Conversely, compound 3 (non-transporter) had no effect on the p38 MAPK activation. The ROS inducer TBHP was shown before to also activate the p38 MAPK pathway (21). To further investigate whether activation of p38 MAPK was related to cell death, A549 cells 
were pretreated with the p38 MAPK inhibitor SB202190 (30 $\mu \mathrm{M})$ for 2 hours before compounds treatment. A significant reversion of cell death induced by compounds 1 and 2 was observed upon inhibitor pre-treatment, which indicates that compounds-mediated cell death is upstream regulated by the p38 MAPK signaling pathway (Fig. 4c).

\section{Therapeutic effect on in vivo mice models.}

To corroborate the anticancer activity of the indole-based tambjamine-analogues seen in several LC cell lines and patient-derived PC, we evaluated the therapeutic effect of compounds 1 and 2 in established DMS53 human small cell lung carcinoma growing subcutaneous and orthotopically in nude mice models. The DMS53 cell line was selected for their ability to grow well in the flank (for subcutaneous model) and in the lung (for orthotopic model) after cell inoculation. Mice bearing DMS53 xenografts were treated with compounds 1 or 2 (6 mg/kg in saline with $7.5 \%$ of DMSO/0.8\% Tween-80) or vehicle control (7.5\% of DMSO/0.8\% Tween-80) in alternating days during 20 days. As shown in Figures $5 a$ and 5b, compound 2 produced a significant decrease in tumor growth in subcutaneous models. Furthermore, treatment with compound 2 significantly $(p<0.05)$ reduced LC tumor progression in the orthotopic lung model (Fig. 5c). Importantly, as a single agent the treatment with compounds 1 or 2 did not produce any obvious toxicity (hepatotoxicity and nephrotoxicity, Fig. 5d), diarrhea or significant body weight loss (Supplementary Fig. S42). 


\section{Discussion}

Chemotherapeutic agents based on natural compounds are a potent source of anti-cancer drugs. Tambjamine analogues of natural compounds tambjamines have already demonstrated a good pharmacological activity and to have remarkable anti-cancer effects $(6,7,11-13)$. In this study, we analyzed the cytotoxic effect and consequent induced cell death and elucidated the molecular mechanism of action of novel synthesized indole-based tambjamine analogues in LC cells. We have observed a significant cytotoxic effect (less than $10 \mu \mathrm{M}$ at $24 \mathrm{~h}$ ) of the selected compounds in several $\mathrm{LC}$ cell lines. Moreover, the selected compounds showed $\mathrm{IC}_{50}$ values remarkably lower than cisplatin, the main chemotherapeutic agent used in the treatment of LC patients.

We have recently demonstrated how tambjamine analogues are able to alter the plasma membrane potential and decrease the intracellular pH in the A549 lung adenocarcinoma cell line (13). Changes in the ion homeostasis of the plasma membrane potential have already been associated with apoptosis-induced cell death in several cell types (S49, Jurkat, HL60, and thymocytes) $(22,23)$. These observations, in addition to our transcriptomic results and the failure to arrest cell cycle, led us to further investigate the molecular events related to the apoptotic process after cell treatment with these compounds.

The mitochondrial apoptotic pathway is closely regulated by the dynamic equilibrium of a group of proteins belonging to the Bcl-2 family with pro-apoptotic (e.g. Bax and Bak) and anti-apoptotic (e.g. $\mathrm{Bcl}-2$ and $\mathrm{Mcl}-1$ ) functions (24-26). The disruption of this equilibrium leads to the mitochondrial outer membrane permeabilization (MOMP) with the consequent release of cytochrome-c from the mitochondria and activation of initiator caspase-9. The initiator caspase-9 then activates the effector

caspase-3 that cleaves regulatory and structural molecules (e.g. poly ADP ribose polymerase, PARP), culminating in the death of the cell $(24,26)$. In this study, the activation of the intrinsic mitochondrial 
pathway and the cleavage of pro-caspase- 9 and pro-caspase- 3 , as well as their substrate PARP were observed in LC cells. In addition, the unbalance of the apoptotic pairs (Bcl-2/Bax and Mcl-1/Bak) towards the pro-apoptotic proteins unveils the final fate of these cells (27). In our system, the expression levels of pro-survival members of the $\mathrm{Bcl}-2$ family ( $\mathrm{Bcl}-2$ and $\mathrm{Mcl}-1)$ decreased in a dosedependent manner and the pro-apoptotic proteins (Bax and Bak) increased after the treatment. In terms of $\mathrm{Bcl}-2$, this decrease further corroborates our transcriptomic results and shows that this alteration is occurring at the gene level. The inhibitor of apoptosis proteins (IAPs, e.g. XIAP and survivin) are proteins that maintain the small leakages of cytochrome-c or small activations of death receptors controlled by inhibiting the initiator and effector caspases. Survivin is a member of the IAP family that prevents mitochondrial-dependent apoptosis through the inhibition of caspases-9 and -3 by direct or indirect binding $(24,28)$. Interestingly, after compounds treatment we have seen a down-regulation of survivin, at both the transcript and protein levels, which also contributes to the triggering of the apoptotic cell death. Furthermore, using a pan-caspase inhibitor, we were able to significantly reverse the cytotoxic effect of our compounds. These results clearly indicate that compounds induce cell death through apoptosis by activating apoptosis-related proteins (caspases-9 and -3 ) and decreasing the levels of anti-apoptotic proteins ( $\mathrm{Bcl}-2, \mathrm{Mcl}-1$ and survivin).

The ionophoric activity of tambjamine analogues has an impact on cellular ion homeostasis and intracellular $\mathrm{pH}$ levels (13), thus, it is feasible that their actions will produce a cellular stress. Several recent works have shown that unbalanced ionic homeostasis is deeply involved in ROS generation (29-31). In our hands, treatment with the selected compounds induced a clear ROS production and this could be mediated by the anion transport since our previous work has shown that these compounds also induce cellular acidification (13). Conversely, the non-transporter compound neither produced $\mathrm{pH}$ changes nor generated ROS. 
The mitogen-activated protein kinases (MAPKs) are crucial signaling players in the integration of stress signals and their conversion to cellular responses (32). One major MAPK signaling pathway that is important for detecting cellular stress, including intracellular acidosis and ROS, is the p38 MAPK cascade (32-34). The p38 MAPK-mediated apoptosis leads to caspase activation $(35,36)$, and several chemotherapeutic drugs have been shown to promote this cascade in order to produce apoptotic cancer cell death (36-42). Moreover, reversion of the apoptotic-mediated cell death produced by chemotherapeutic drugs that de-polymerize (nocodazole, vincristine and vinblastine) or stabilize (taxol) microtubules, was achieved by using p38 MAPK-specific inhibitors (e.g. SB203580 and SB202190). Likewise, genotoxic agents (cisplatin and oxaliplatin) and topoisomerase II inhibitors (doxorubicin) were also able to activate p38 MAPK route $(43,44)$. Nowadays, a dual-role of this route is known with the action as tumor suppressor or tumor promoter largely depending on the type of cancer and tumor stage (45). Interestingly, our results have shown a robust p38 MAPK activation and using the specific p38 MAPK inhibitor, SB202190, we were able to significantly restore cell viability. Reversion of cell death using the pan-caspase inhibitor or the stress kinase inhibitor is very similar, which foresight that our compounds mediate cell death by apoptosis mainly through the p38 MAPK route.

On the other hand, several transcription factors have been implicated in the regulation of both antiapoptotic proteins, survivin and Bcl-2: Sp1, p53, NF-KB and STAT3 $(38,46-48)$. While Sp1, NF-KB and STAT3 are generally implicated in transactivation of $\mathrm{Bcl}-2$ and survivin genes, p53 was shown to be responsible for transcriptional repression of these genes. Moreover, these transcription factors are all substrates of the p38 MAPK signaling pathway (49). In fact, several articles connect p38 MAPK activation with a down-regulation of survivin and Bcl-2 expressions (39, 40, 50-53). Nevertheless, caution should be taken since other pathways are also involved in the activation/repression of these transcription factors and so, crosstalk with other routes is possible. Further experiments are 
underway to understand the regulation of these genes, which have a profound impact in the fate of the cells by apoptosis. Our study has also shown the therapeutic effect of our compounds in a preclinical setting using subcutaneous and orthotopic mouse models for LC, which indicates a good potential for pharmaceutical development.

In conclusion, we have shown the cytotoxic effect of novel indole-based tambjamine analogues towards LC cells in vitro and in vivo. We have also identified several gene-expression profile alterations produced by our compounds in LC cells and have shown that the main molecular route of induced cell death is apoptosis, which might be activated by the p38 MAPK through ROS cellular stress induction. Ultimately, by understanding the mechanism of action through which these naturalbased small molecules mediate their effect in cancer cells will provide a way to improve future studies of drug efficacy and pharmacodynamics, as well as establish drug-response biomarkers and synergistic drug combinations. 


\section{Acknowledgments}

We thank Beatriz Barroso from CCiTUB (Centres Científics i Tecnològics Universitat de Barcelona, Barcelona, Spain) for technical assistance. 


\section{References}

1. Ferlay J, Soerjomataram I, Ervik M, Dikshit R, Eser S, Mathers C, et al. GLOBOCAN 2012 v1.0, Cancer Incidence and Mortality Worldwide: IARC CancerBase No. 11 [Internet]. Lyon, France: International Agency for Research on Cancer. 2013. Available from: http://globocan.iarc.fr, accessed on 20/07/2016.

2. Carrera PM, Ormond M. Current practice in and considerations for personalized medicine in lung cancer: From the patient's molecular biology to patient values and preferences. Maturitas. 2015;82:94-9.

3. Molinski TF, Dalisay DS, Lievens SL, Saludes JP. Drug development from marine natural products. Nat Rev Drug Discov. 2009;8:69-85.

4. Simmons TL, Andrianasolo E, McPhail K, Flatt P, Gerwick WH. Marine natural products as anticancer drugs. Mol Cancer Ther. 2005;4:333-42.

5. Mayer AM, Glaser KB, Cuevas C, Jacobs RS, Kem W, Little RD, et al. The odyssey of marine pharmaceuticals: a current pipeline perspective. Trends Pharmacol Sci. 2010;31:255-65.

6. Carbone M, Irace C, Costagliola F, Castelluccio F, Villani G, Calado G, et al. A new cytotoxic tambjamine alkaloid from the Azorean nudibranch Tambja ceutae. Bioorg Med Chem Lett. 2010;20:2668-70.

7. Gale PA, Perez-Tomas R, Quesada R. Anion transporters and biological systems. Acc Chem Res. 2013;46:2801-13.

8. Llagostera E, Soto-Cerrato V, Montaner B, Perez-Tomas R. Prodigiosin induces apoptosis by acting on mitochondria in human lung cancer cells. Ann N Y Acad Sci. 2003;1010:178-81.

9. Perez-Tomas R, Montaner B, Llagostera E, Soto-Cerrato V. The prodigiosins, proapoptotic drugs with anticancer properties. Biochem Pharmacol. 2003;66:1447-52.

10. Soto-Cerrato V, Llagostera E, Montaner B, Scheffer GL, Perez-Tomas R. Mitochondria-mediated apoptosis operating irrespective of multidrug resistance in breast cancer cells by the anticancer agent prodigiosin. Biochem Pharmacol. 2004;68:1345-52.

11. Iglesias Hernandez P, Moreno D, Javier AA, Torroba T, Perez-Tomas R, Quesada R. Tambjamine alkaloids and related synthetic analogs: efficient transmembrane anion transporters. Chem Commun (Camb). 2012;48:1556-8.

12. Hernando E, Soto-Cerrato V, Cortes-Arroyo S, Perez-Tomas R, Quesada R. Transmembrane anion transport and cytotoxicity of synthetic tambjamine analogs. Org Biomol Chem. 2014;12:1771-8.

13. Soto-Cerrato V, Manuel-Manresa P, Hernando E, Calabuig-Farinas S, Martinez-Romero A, FernandezDuenas $V$, et al. Facilitated Anion Transport Induces Hyperpolarization of the Cell Membrane That Triggers Differentiation and Cell Death in Cancer Stem Cells. J Am Chem Soc. 2015;137:15892-8.

14. Gupta PB, Chaffer CL, Weinberg RA. Cancer stem cells: mirage or reality? Nat Med. 2009;15:1010-2.

15. Shannon P, Markiel A, Ozier O, Baliga NS, Wang JT, Ramage D, et al. Cytoscape: a software environment for integrated models of biomolecular interaction networks. Genome Res. 2003;13:2498-504.

16. Ambrogio C, Carmona FJ, Vidal A, Falcone M, Nieto P, Romero OA, et al. Modeling lung cancer evolution and preclinical response by orthotopic mouse allografts. Cancer Res. 2014;74:5978-88.

17. Ambrogio C, Gomez-Lopez G, Falcone M, Vidal A, Nadal E, Crosetto N, et al. Combined inhibition of DDR1 and Notch signaling is a therapeutic strategy for KRAS-driven lung adenocarcinoma. Nat Med. 2016;22:270-7.

18. Yan R, Yang $\mathrm{Y}$, Zeng $\mathrm{Y}$, Zou G. Cytotoxicity and antibacterial activity of Lindera strychnifolia essential oils and extracts. J Ethnopharmacol. 2009;121:451-5.

19. Zhan $M, Q u ~ Q$, Wang $G$, Zhou $H$. Let-7c sensitizes acquired cisplatin-resistant A549 cells by targeting ABCC2 and BCl-XL. Pharmazie. 2013;68:955-61.

20. Mueller S, Schittenhelm M, Honecker F, Malenke E, Lauber K, Wesselborg S, et al. Cell-cycle progression and response of germ cell tumors to cisplatin in vitro. Int J Oncol. 2006;29:471-9.

21. Yang Y, Liu X, Huang J, Zhong Y, Mao Z, Xiao H, et al. Inhibition of p38 mitogen-activated protein kinase phosphorylation decrease tert-butyl hydroperoxide-induced apoptosis in human trabecular meshwork cells. Mol Vis. 2012;18:2127-36. 
22. Dallaporta B, Marchetti P, de Pablo MA, Maisse C, Duc H-T, Métivier D, et al. Plasma membrane potential in thymocyte apoptosis. The Journal of Immunology. 1999;162:6534-42.

23. Bortner CD, Gómez-Angelats $\mathrm{M}$, Cidlowski JA. Plasma membrane depolarization without repolarization is an early molecular event in anti-Fas-induced apoptosis. J Biol Chem. 2001;276:4304-14.

24. Ghobrial IM, Witzig TE, Adjei AA. Targeting apoptosis pathways in cancer therapy. CA Cancer J Clin. 2005;55:178-94.

25. Wong RS. Apoptosis in cancer: from pathogenesis to treatment. J Exp Clin Cancer Res. 2011;30:87.

26. Bai L, Wang S. Targeting apoptosis pathways for new cancer therapeutics. Annu Rev Med. 2014;65:139-55.

27. Czabotar PE, Lessene G, Strasser A, Adams JM. Control of apoptosis by the BCL-2 protein family: implications for physiology and therapy. Nat Rev Mol Cell Biol. 2014;15:49-63.

28. Pavlyukov MS, Antipova NV, Balashova MV, Vinogradova TV, Kopantzev EP, Shakhparonov MI. Survivin monomer plays an essential role in apoptosis regulation. J Biol Chem. 2011;286:23296-307.

29. Ko SK, Kim SK, Share A, Lynch VM, Park J, Namkung W, et al. Synthetic ion transporters can induce apoptosis by facilitating chloride anion transport into cells. Nat Chem. 2014;6:885-92.

30. Saha T, Hossain MS, Saha D, Lahiri M, Talukdar P. Chloride-Mediated Apoptosis-Inducing Activity of Bis(sulfonamide) Anionophores. J Am Chem Soc. 2016;138:7558-67.

31. Zhao $W$, Lu M, Zhang $Q$. Chloride intracellular channel 1 regulates migration and invasion in gastric cancer by triggering the ROS-mediated p38 MAPK signaling pathway. Mol Med Rep. 2015;12:8041-7.

32. Wagner EF, Nebreda AR. Signal integration by JNK and p38 MAPK pathways in cancer development. Nat Rev Cancer. 2009;9:537-49.

33. Zarubin T, Han J. Activation and signaling of the p38 MAP kinase pathway. Cell Res. 2005;15:11-8.

34. Son Y, Kim S, Chung HT, Pae HO. Reactive oxygen species in the activation of MAP kinases. Methods Enzymol. 2013;528:27-48.

35. Tsuchiya T, Tsuno NH, Asakage M, Yamada J, Yoneyama S, Okaji Y, et al. Apoptosis induction by p38 MAPK inhibitor in human colon cancer cells. Hepatogastroenterology. 2008;55:930-5.

36. Li QC, Liang Y, Tian Y, Hu GR. Arctigenin induces apoptosis in colon cancer cells through ROS/p38MAPK pathway. J BUON. 2016;21:87-94.

37. Olson JM, Hallahan AR. p38 MAP kinase: a convergence point in cancer therapy. Trends Mol Med. 2004;10:125-9.

38. Bodur C, Kutuk O, Karsli-Uzunbas G, Isimjan TT, Harrison P, Basaga H. Pramanicin analog induces apoptosis in human colon cancer cells: critical roles for Bcl-2, Bim, and p38 MAPK signaling. PLoS One. 2013;8:e56369.

39. Hsiao PW, Chang CC, Liu HF, Tsai CM, Chiu TH, Chao Jl. Activation of p38 mitogen-activated protein kinase by celecoxib oppositely regulates survivin and gamma-H2AX in human colorectal cancer cells. Toxicol Appl Pharmacol. 2007;222:97-104.

40. Liu HF, Hu HC, Chao Jl. Oxaliplatin down-regulates survivin by p38 MAP kinase and proteasome in human colon cancer cells. Chem Biol Interact. 2010;188:535-45.

41. Ahn J, Won M, Choi JH, Kim YS, Jung CR, Im DS, et al. Reactive oxygen species-mediated activation of the Akt/ASK1/p38 signaling cascade and p21(Cip1) downregulation are required for shikonin-induced apoptosis. Apoptosis : an international journal on programmed cell death. 2013;18:870-81.

42. Pan J, Chang $Q$, Wang X, Son $Y$, Zhang Z, Chen G, et al. Reactive oxygen species-activated Akt/ASK1/p38 signaling pathway in nickel compound-induced apoptosis in BEAS 2B cells. Chem Res Toxicol. 2010;23:568-77.

43. Hernandez Losa J, Parada Cobo C, Guinea Viniegra J, Sanchez-Arevalo Lobo VJ, Ramon y Cajal S, Sanchez-Prieto R. Role of the p38 MAPK pathway in cisplatin-based therapy. Oncogene. 2003;22:3998-4006.

44. Fujie Y, Yamamoto H, Ngan CY, Takagi A, Hayashi T, Suzuki R, et al. Oxaliplatin, a potent inhibitor of survivin, enhances paclitaxel-induced apoptosis and mitotic catastrophe in colon cancer cells. Jpn J Clin Oncol. 2005;35:453-63. 
45. Garcia-Cano J, Roche O, Cimas FJ, Pascual-Serra R, Ortega-Muelas M, Fernandez-Aroca DM, et al. p38MAPK and Chemotherapy: We Always Need to Hear Both Sides of the Story. Front Cell Dev Biol. 2016;4:69.

46. Chen X, Duan N, Zhang C, Zhang W. Survivin and Tumorigenesis: Molecular Mechanisms and Therapeutic Strategies. J Cancer. 2016;7:314-23.

47. Li F, Altieri DC. Transcriptional analysis of human survivin gene expression. Biochem J. 1999;344:305-

11.

48. Carpenter RL, Lo HW. STAT3 Target Genes Relevant to Human Cancers. Cancers (Basel). 2014;6:897925.

49. Cuadrado A, Nebreda AR. Mechanisms and functions of p38 MAPK signalling. Biochem J. 2010;429:403-17.

50. Cao W, Xie YH, Li XQ, Zhang XK, Chen YT, Kang R, et al. Burn-induced apoptosis of cardiomyocytes is survivin dependent and regulated by PI3K/Akt, p38 MAPK and ERK pathways. Basic Res Cardiol. 2011;106:1207-20.

51. Changchien JJ, Chen YJ, Huang CH, Cheng TL, Lin SR, Chang LS. Quinacrine induces apoptosis in human leukemia K562 cells via p38 MAPK-elicited BCL2 down-regulation and suppression of ERK/c-Junmediated BCL2L1 expression. Toxicol Appl Pharmacol. 2015;284:33-41.

52. Chen YJ, Liu WH, Kao PH, Wang JJ, Chang LS. Involvement of p38 MAPK- and JNK-modulated expression of $\mathrm{Bcl}-2$ and Bax in Naja nigricollis CMS-9-induced apoptosis of human leukemia K562 cells. Toxicon. 2010;55:1306-16.

53. Hui K, Yang Y, Shi K, Luo H, Duan J, An J, et al. The p38 MAPK-regulated PKD1/CREB/Bcl-2 pathway contributes to selenite-induced colorectal cancer cell apoptosis in vitro and in vivo. Cancer Lett. 2014;354:189-99. 


\section{Tables}

Table 1. Genes modulated after indole-based tambjamine analogues treatment. The A549 lung adenocarcinoma cell line was treated with the $\mathrm{IC}_{50}$ values of compounds 1 or 2 and the expression of 84 genes related to cancer drug targets was analyzed by qRT-PCR (QIAGEN RT2 Profiler Array). Foldregulation values of the most altered genes are presented below.

\begin{tabular}{|c|c|c|c|c|c|c|}
\hline & \multirow{2}{*}{ Protein Name } & \multicolumn{2}{|c|}{1} & \multicolumn{2}{|c|}{2} & \multirow{2}{*}{ Functional Gene Grouping } \\
\hline & & $6 h$ & $16 \mathrm{~h}$ & $6 h$ & $16 h$ & \\
\hline \multicolumn{7}{|c|}{ Down-regulated } \\
\hline $\mathrm{ABCC} 1$ & ATP-binding cassette, sub-family $C$, member 1 & -1.11 & -1.61 & -1.48 & -2.32 & Drug Metabolism \\
\hline AURKA & Aurora kinase $\mathrm{A}$ & -2.53 & -2.37 & -2.69 & -4.13 & Protein Kinases \\
\hline AURKB & Aurora kinase B & -1.96 & -2.43 & -2.16 & -5.03 & Protein Kinases \\
\hline $\mathrm{BCL} 2$ & B-cell CLL/lymphoma 2 & -2.17 & 1.10 & -1.83 & -1.90 & Apoptosis \\
\hline BIRC5 & Baculoviral IAP repeat containing 5, Survivin & -2.08 & -2.37 & -2.53 & -4.72 & Apoptosis \\
\hline CDC25A & Cell division cycle 25 homolog A & -2.30 & -1.75 & -3.72 & -4.11 & Cell Cycle \\
\hline CDK1 & Cyclin-dependent kinase 1 & -1.97 & -2.71 & -2.41 & -3.94 & Cell Cycle \\
\hline CDK2 & Cyclin-dependent kinase 2 & -1.81 & -1.73 & -1.24 & -3.21 & Cell Cycle \\
\hline ERBB3 & Receptor tyrosine-protein kinase erbB-3, HER3 & -2.42 & -1.35 & -2.81 & -2.18 & Growth Factors \& Receptors \\
\hline ESR2 & Estrogen receptor 2 & -1.65 & -1.20 & -2.87 & -1.78 & Hormone Receptors \\
\hline FIGF & C-fos induced growth factor, VEGFD & -2.25 & -1.65 & -3.57 & -3.74 & Growth Factors \& Receptors \\
\hline HDAC8 & Histone deacetylase 8 & -1.87 & 1.11 & -1.52 & -2.01 & Histone Deacetylases \\
\hline HSP90AA1 & Heat shock protein 90kDa alpha, member 1 & -1.84 & -1.62 & -1.43 & -2.47 & Heat Shock Proteins \\
\hline IGF1 & Insulin-like growth factor 1 , Somatomedin C & -4.89 & -17.47 & -3.48 & -42.18 & Growth Factors \& Receptors \\
\hline NTN3 & Netrin 3 & -1.81 & -2.11 & -2.85 & -1.17 & Structural Protein \\
\hline PARP2 & Poly (ADP-ribose) polymerase 2 & -1.29 & -1.63 & -1.63 & -2.20 & Poly ADP-Ribose Polymerases \\
\hline PDGFRA & Platelet-derived growth factor receptor alpha & -3.55 & -1.59 & 1.10 & -2.48 & Growth Factors \& Receptors \\
\hline PLK1 & Polo-like kinase 1 & -2.24 & -2.46 & -2.36 & -4.13 & Protein Kinases \\
\hline PLK4 & Polo-like kinase 4 & -1.36 & -2.51 & -1.20 & -2.02 & Protein Kinases \\
\hline TOP2A & Topoisomerase (DNA) II alpha 170kDa & -2.06 & -2.78 & -2.09 & -4.30 & Topoisomerases, Type II \\
\hline \multicolumn{7}{|c|}{ Up-regulated } \\
\hline CTSL1 & Cathepsin L1 & 1.30 & 1.92 & 2.03 & 2.07 & Cathepsins \\
\hline CTSS & Cathepsin S & 1.24 & 2.69 & 1.71 & 2.08 & Cathepsins \\
\hline HSP90B1 & Heat shock protein 90kDa beta, member 1 & 2.00 & 1.95 & 1.63 & 1.97 & Heat Shock Proteins \\
\hline NFKB1 & Nuclear factor NF-kappa-B p105 subunit & 3.88 & 1.23 & 3.14 & -1.01 & Transcription Factors \\
\hline PRKCE & Protein kinase $C$, epsilon & 1.52 & -1.04 & 2.53 & 1.95 & Protein Kinases \\
\hline PTGS2 & Prostaglandin-endoperoxide synthase $2, \mathrm{COX}-2$ & 6.46 & -1.23 & 19.29 & 2.90 & Drug Metabolism \\
\hline
\end{tabular}




\section{Figure Legends}

Figure 1. Effect of indole-based tambjamine analogues on cell survival. a) Single point screening of synthetic tambjamine analogues 1-11 (10 $\mu \mathrm{M})$ on a panel of LC cell lines (A549, DMS53, SW900 and $\mathrm{H} 460$ ). b) IC $\mathrm{C}_{50}$ values of selected compounds (1 and 2) on LC cell lines and LC patient-derived primary cultures (PC\#8 and PC\#13). For comparison purposes, cisplatin (CDDP) was used as the standard clinical chemotherapeutic agent. Viability was measured using the MTT assay after 24 hours of treatment. Results were obtained from at least three independent experiments, and bar represents the mean \pm S.D.

Figure 2. Indole-based tambjamine analogues effect on cellular signaling pathways. A directednetwork of differentially expressed genes after compounds 1 or 2 treatment in A549 cells was constructed from the RT-PCR array results and data extracted from Gene Network Central (GNCPro).

Figure 3. Analysis of the apoptotic pathway after indole-based tambjamine analogues exposure. a) After 24 hours treatment with the $I C_{25}, I C_{50}$ and $I C_{75}$ values of compounds 1 or 2 , the expression of several apoptotic markers was analyzed by Western blot in A549 cell line. b, c) Bcl-2 family protein complexes (Bak/Mcl-1 and Bax/Bcl-2, respectively) ratios are represented in bar graphs. d) A549 cells were treated with $20 \mu \mathrm{M}$ of Z-VAD-FMK for 2 hours followed by compound 1 or 2 for 6 hours. Cell viability was measured using the flow cytometry based MUSE ${ }^{\mathrm{TM}}$ Cell Analyzer Kit. Results were obtained from at least three independent experiments. Bars represent the mean \pm S.D. Statistically significant results are indicated as $* p<0.05,{ }^{* *} p<0.01$ and ${ }^{* * *} p<0.001$

Figure 4. Indole-based tambjamine analogues induce ROS, causing cellular stress by p38 MAPK activation. a) A549 cells were treated with compounds 1, 2 or 3 (negative control) for 6 hours or with TBHP (positive control) for 2 hours. ROS formation was measured using the flow cytometry based MUSE ${ }^{\mathrm{TM}}$ Oxidative Stress kit. b) Phosphorylation levels of the stress kinase p38 MAPK were assessed by Western blot in $A 549$ cells exposed to the $I C_{25}, I C_{50}$ and $I C_{75}$ values of compounds 1,2 or 3 for 24 h. c) A549 cells were treated with $30 \mu \mathrm{M}$ of SB20219 for 2 hours followed by compounds 1 or 2 for 6 hours. Cell viability was measured using the flow cytometry based MUSE ${ }^{\mathrm{TM}}$ Cell Analyzer Kit. Results 
were obtained from at least three independent experiments. Bars represent the mean \pm S.D. Statistically significant results are indicated as $* p<0.05, * * p<0.01$ and $* * * p<0.001$.

Figure 5. Therapeutic effect of indole-based tambjamine-analogues in LC in vivo mouse models. a) Growth curve of subcutaneous tumor volumes after compound treatment show significantly differences between compound 2 vs control. b) The weight of subcutaneous tumors compared with control animals is significantly lower after compound 2 treatment. c) Compound 2 treatment reduce significantly the tumor weight in orthotopic DMS53 mouse xenografts model. Statistical analysis was performed using the nonparametric tests. ${ }^{*} p<0.05$ and ${ }^{* *} p<0.01$. d) Representative illustrations of liver and kidney histology using hematoxilin-eosin staining, at 100x and 400x magnifications (scale bars correspond to $200 \mu \mathrm{m}$ and $50 \mu \mathrm{m}$, respectively). Histopathological examination of mice liver and kidney detected no obvious pathological changes after compound 1 or 2 treatment. 
Figure 1

a) $=\mathrm{A} 549=\mathrm{DMS53}=\mathrm{SW900} \approx \mathrm{H} 460$

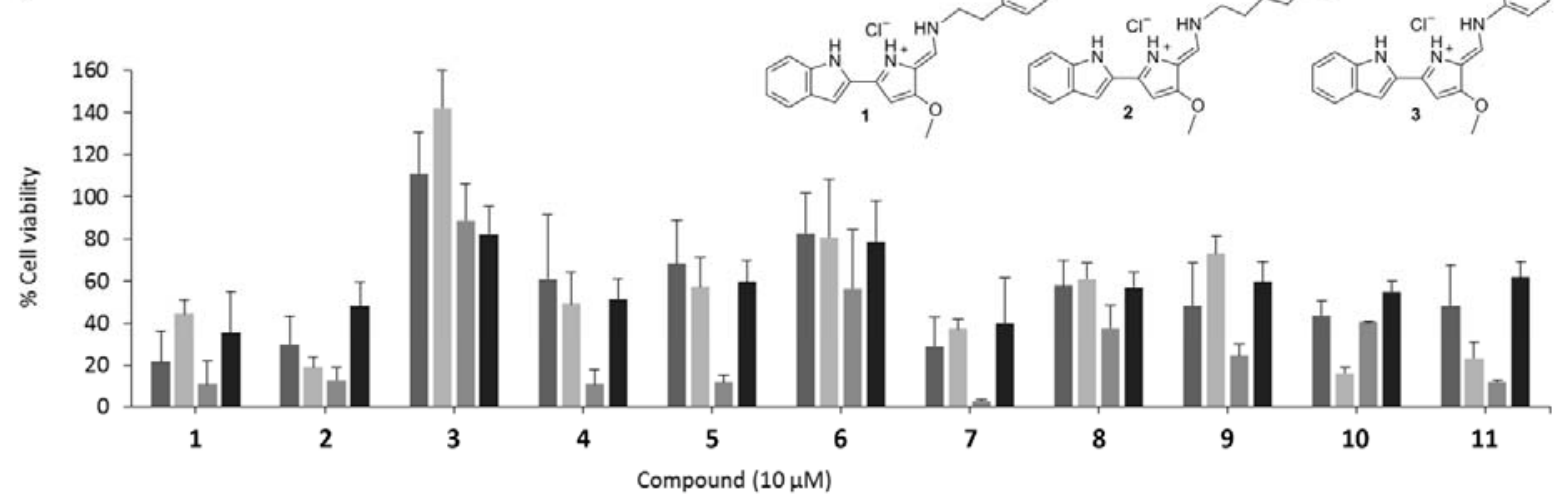

b)

\begin{tabular}{|c|c|c|c|c|c|c|}
\hline & A549 & DMS53 & SW900 & H460 & PCH8 & PC\#13 \\
\hline 1 & $10.66 \pm 0.68$ & $8.04 \pm 0.45$ & $8.67 \pm 0.06$ & $8.37 \pm 0.24$ & $4.04 \pm 1.29$ & $4.34 \pm 0.25$ \\
\hline 2 & $7.61 \pm 0.23$ & $6.46 \pm 0.52$ & $7.55 \pm 0.89$ & $7.29 \pm 0.29$ & $3.34 \pm 0.86$ & $4.03 \pm 0.19$ \\
\hline CDDP & IC $>200$ & $107.9 \pm 31.16$ & $49.4 \pm 10.56$ & $74.2 \pm 6.72$ & $49.28 \pm 9.88$ & $79.57 \pm 9.37$ \\
\hline
\end{tabular}




\section{Figure 2}

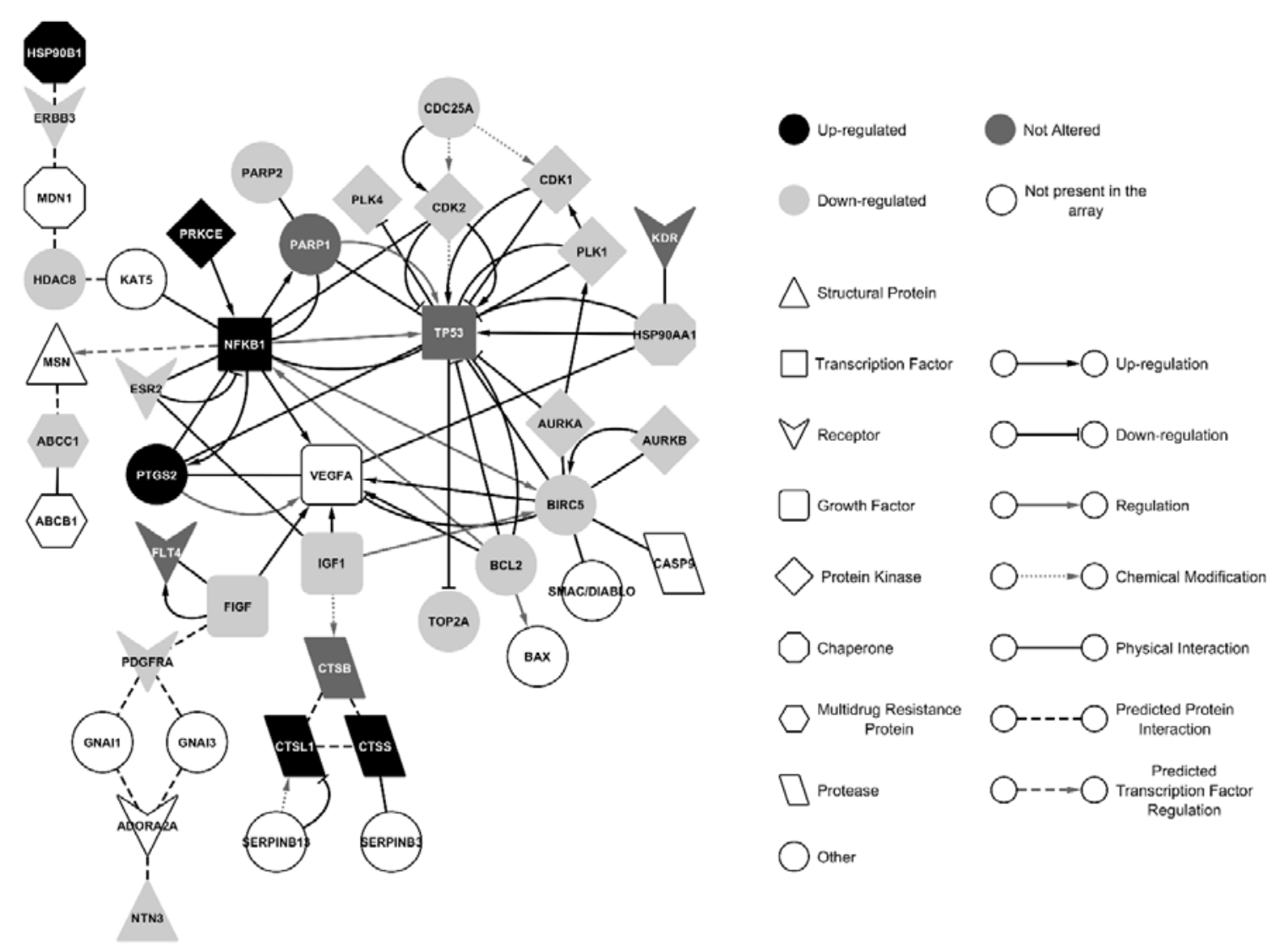


Figure 3

a)

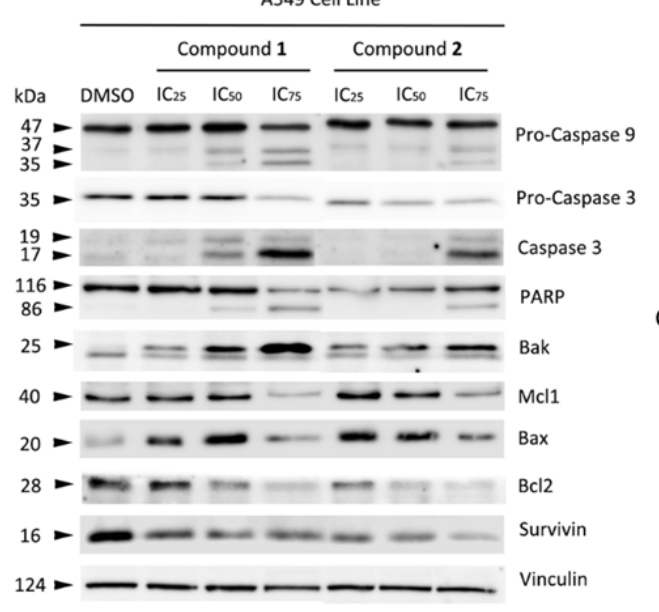

b)

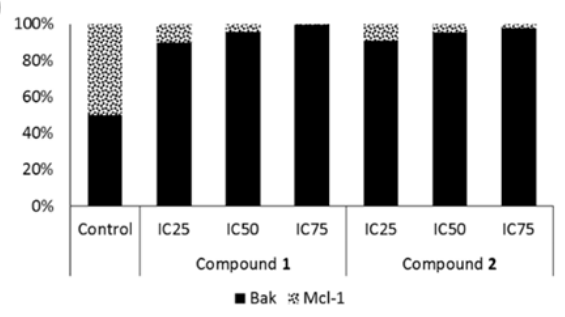

c)

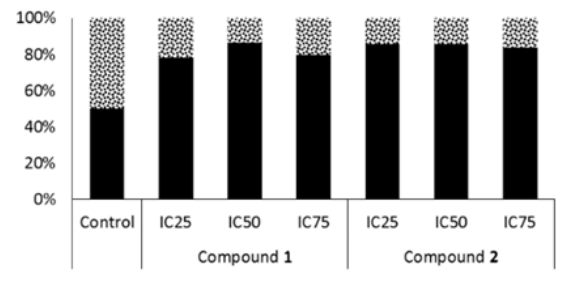

- Bax \& Bcl-2

d)

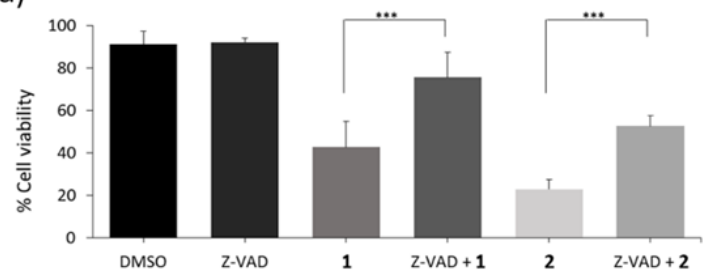


Figure 4

a)

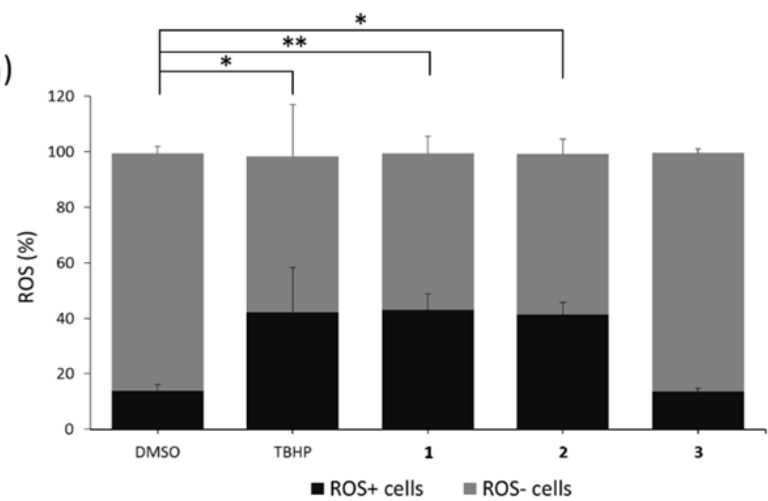

b)
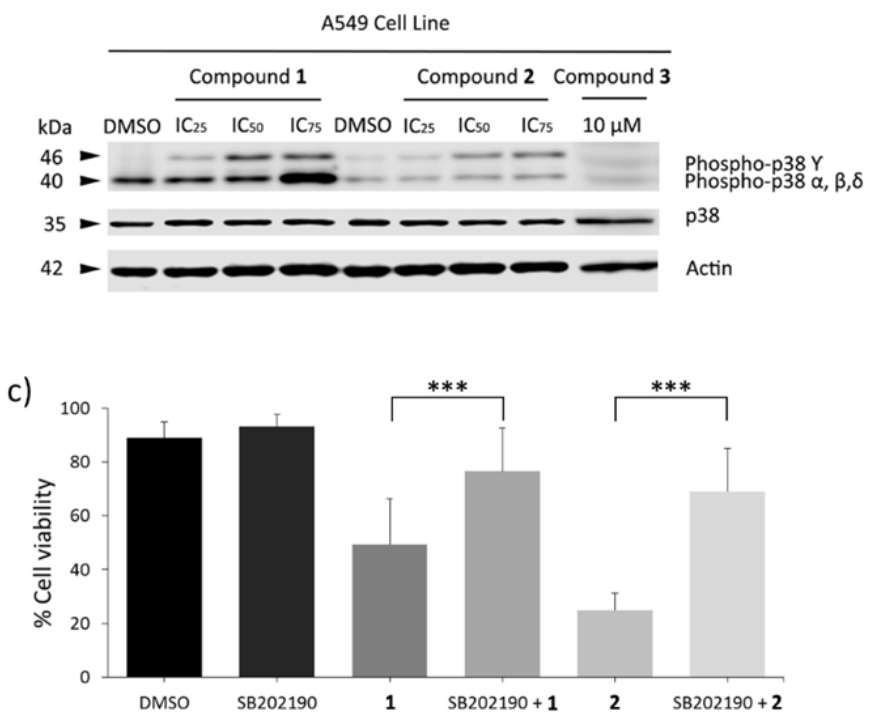


\section{Figure 5}

a) Subcutaneous model

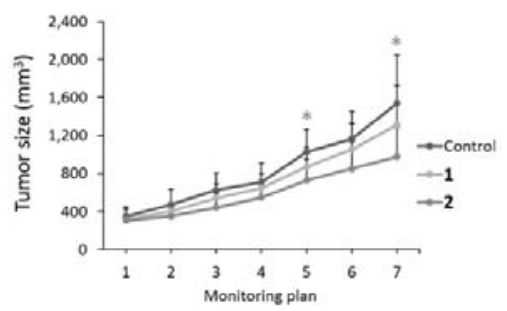

d)
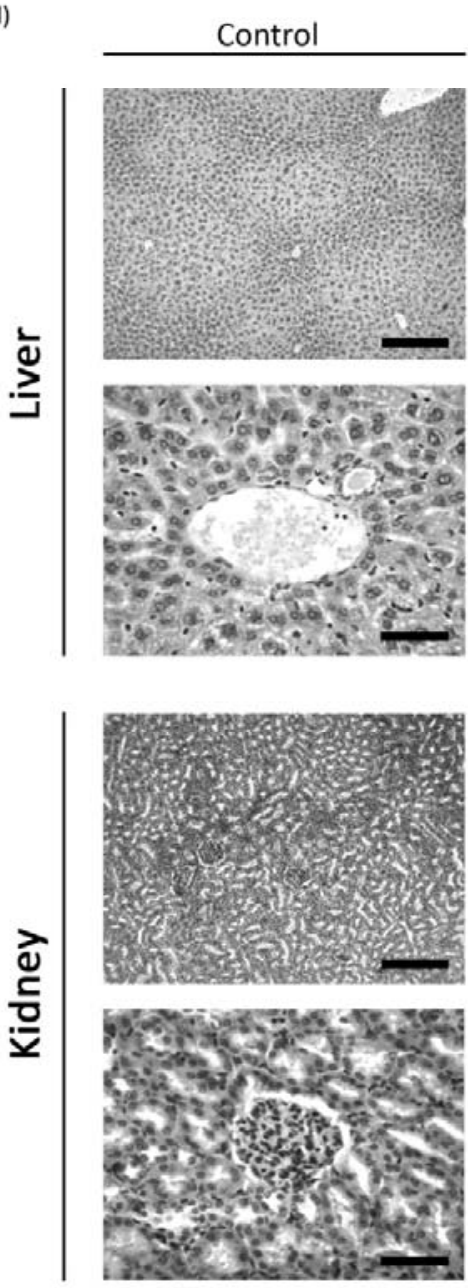

b) Subcutaneous model

c) Orthotopic model
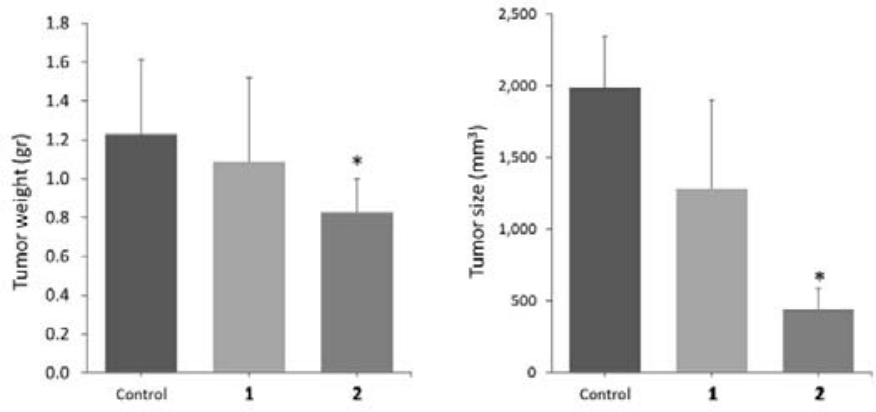

1

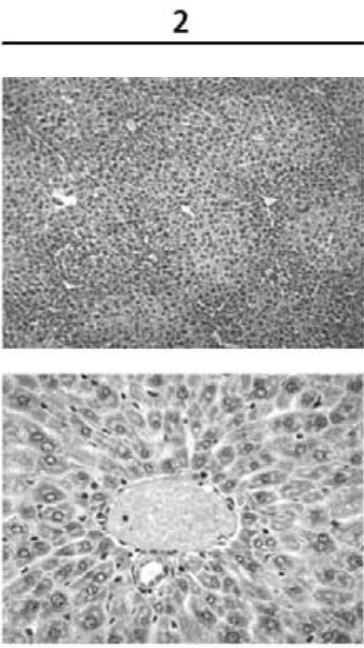

$100 x$

$400 x$

$100 x$
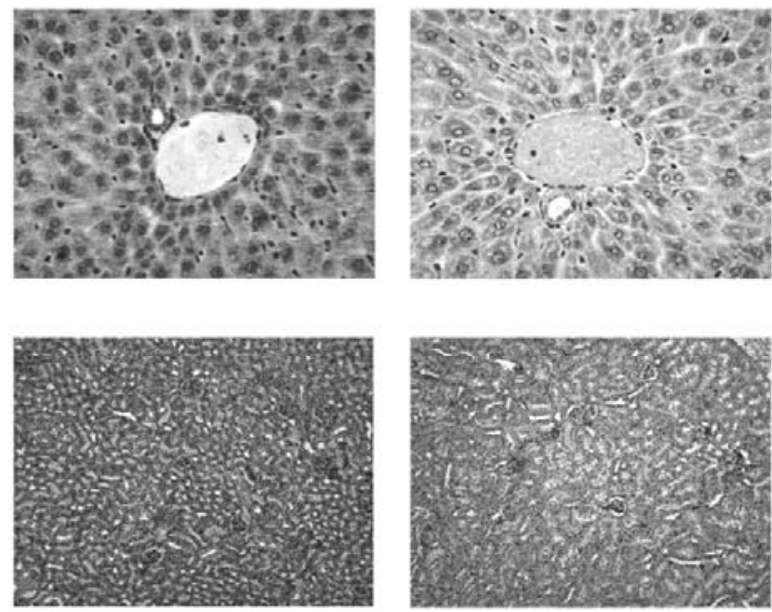

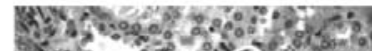

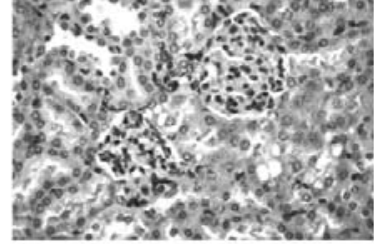


Fig. 1

a) $\quad \mathrm{A} 549 \square \mathrm{DMS53} \square \mathrm{SW} 900 \square \mathrm{H} 460$

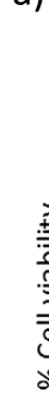

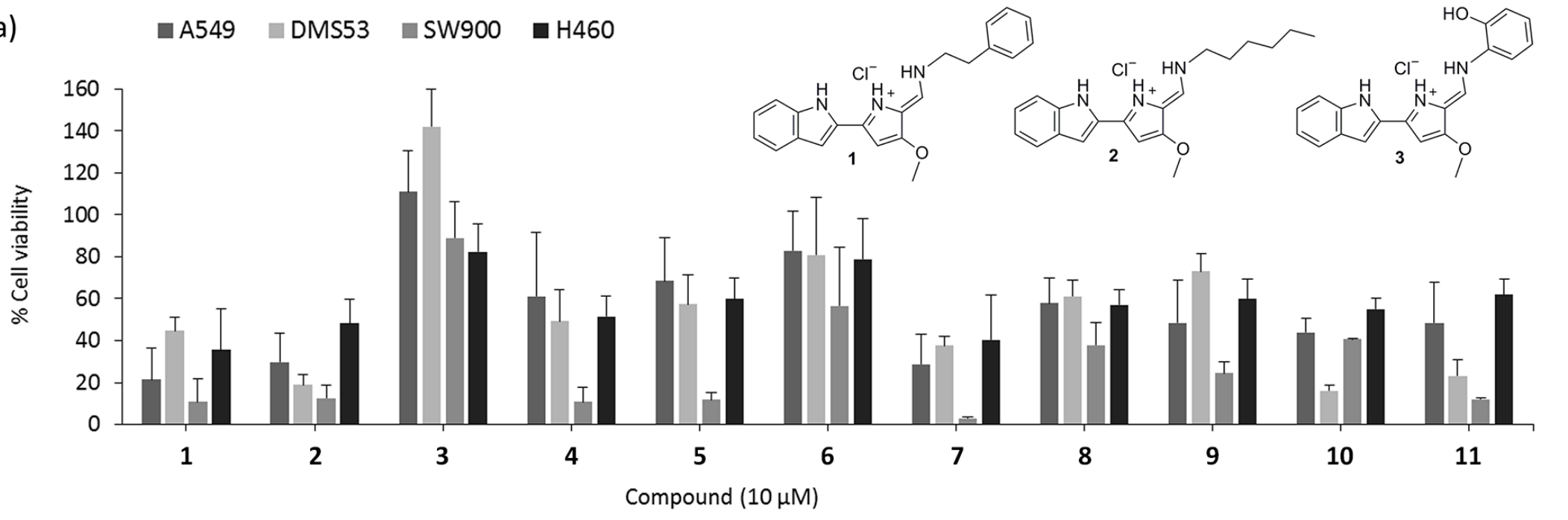

b)

\begin{tabular}{c|c|c|c|c|c|c}
\cline { 2 - 6 } & A549 & DMS53 & SW900 & H460 & \multicolumn{1}{|c|}{ PC\#8 } & \multicolumn{1}{c}{ PC\#13 } \\
\cline { 1 - 5 } $\mathbf{1}$ & $10.66 \pm 0.68$ & $8.04 \pm 0.45$ & $8.67 \pm 0.06$ & $8.37 \pm 0.24$ & $4.04 \pm 1.29$ & $4.34 \pm 0.25$ \\
$\mathbf{2}$ & $7.61 \pm 0.23$ & $6.46 \pm 0.52$ & $7.55 \pm 0.89$ & $7.29 \pm 0.29$ & $3.34 \pm 0.86$ & $4.03 \pm 0.19$ \\
\cline { 1 - 5 } CDDP & IC $>200$ & $107.9 \pm 31.16$ & $49.4 \pm 10.56$ & $74.2 \pm 6.72$ & $49.28 \pm 9.88$ & $79.57 \pm 9.37$ \\
\hline
\end{tabular}



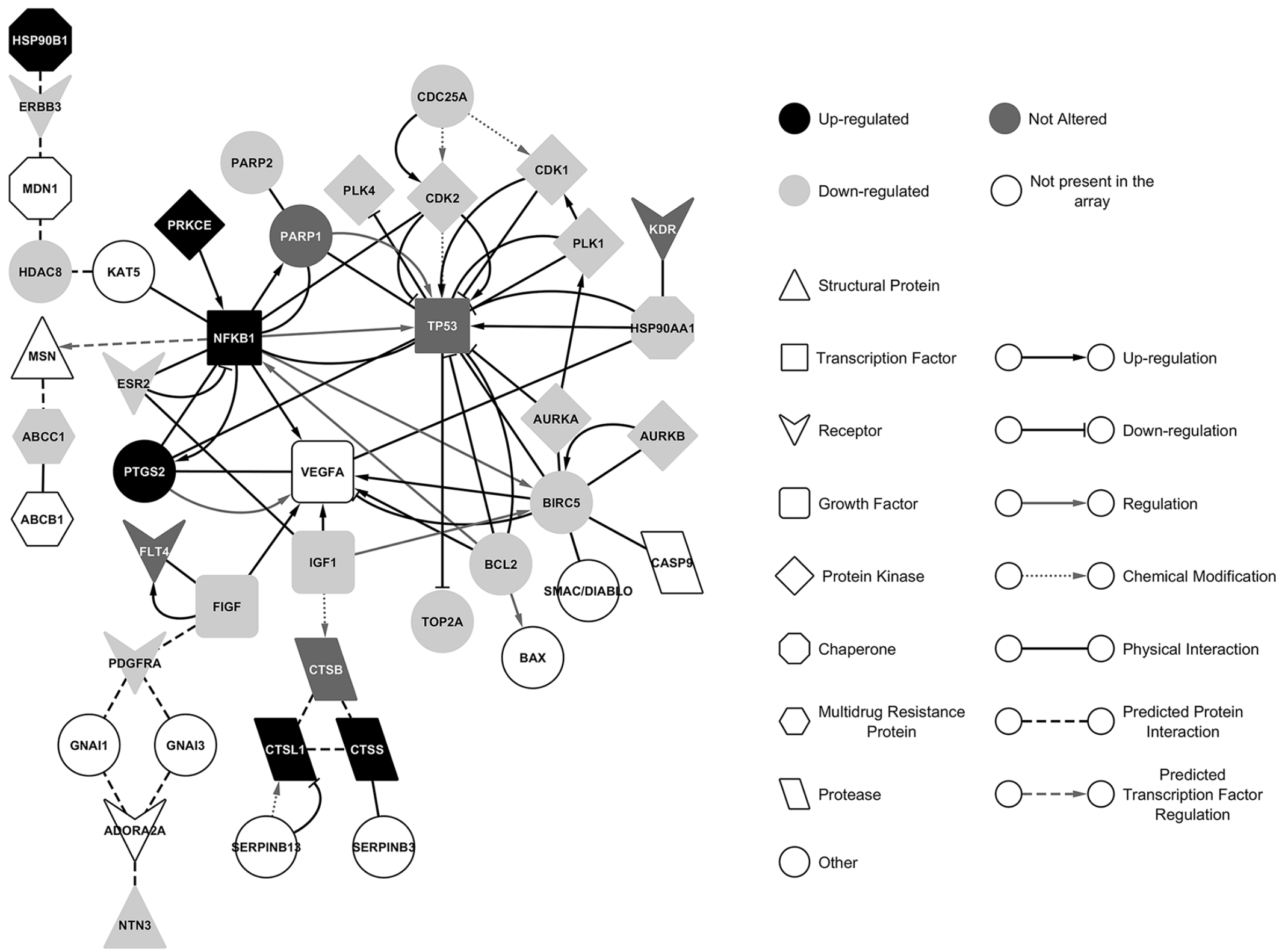
a)

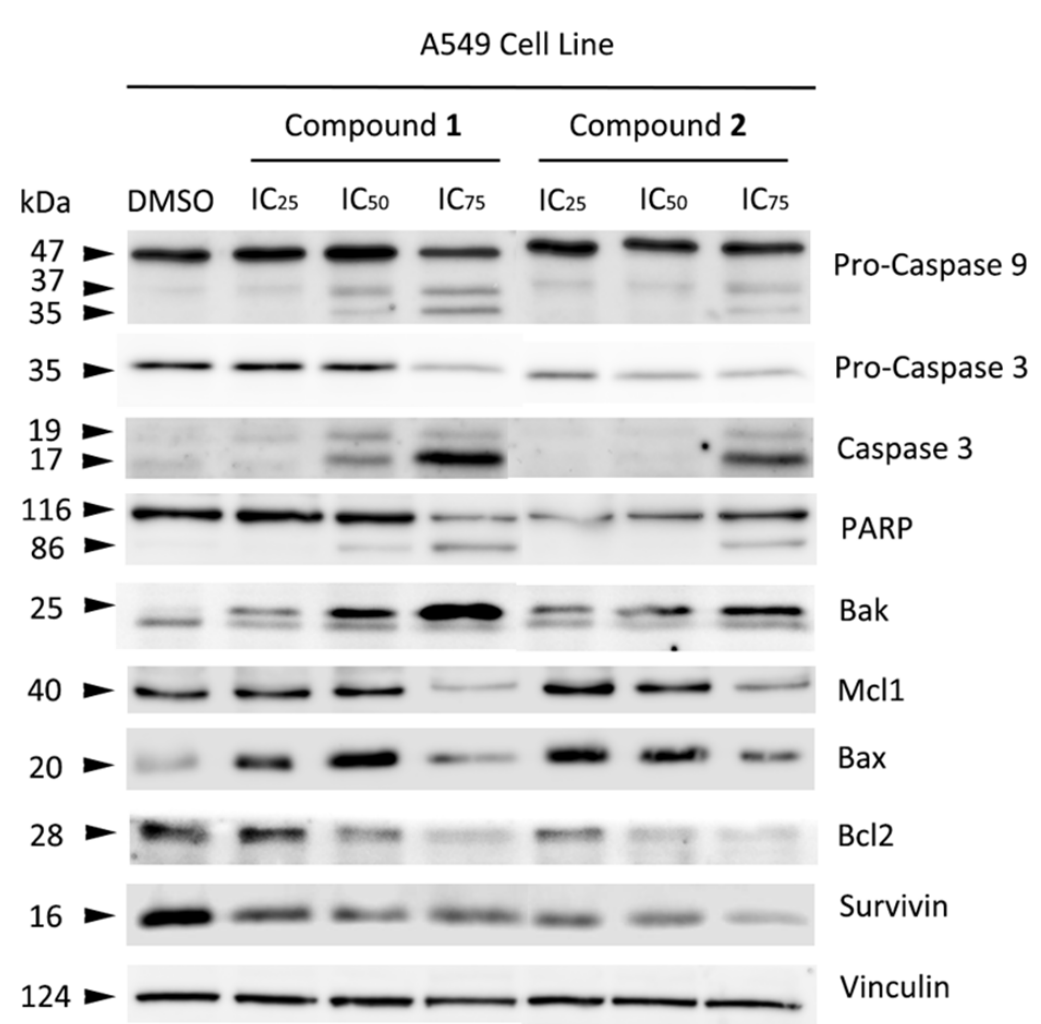

b)

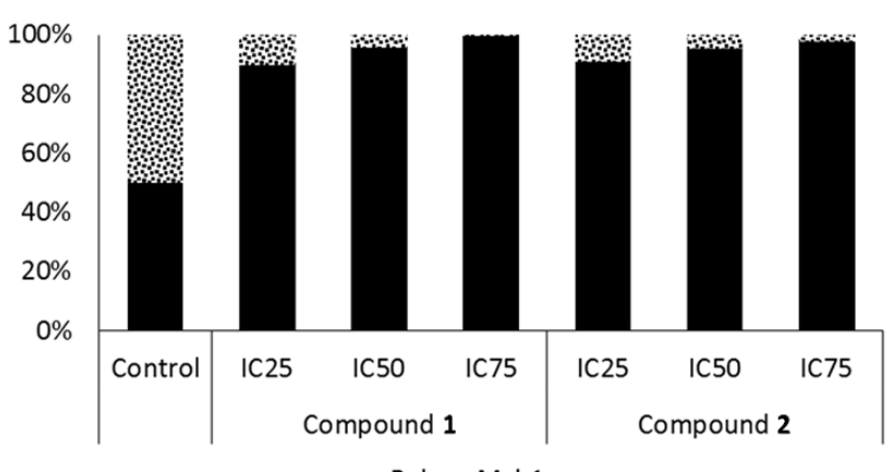

c)

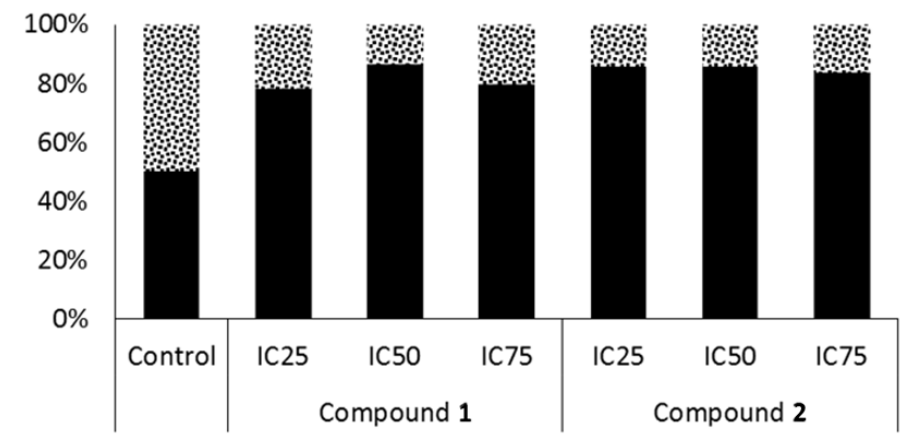

- Bax $\therefore \mathrm{Bcl}-2$

d)

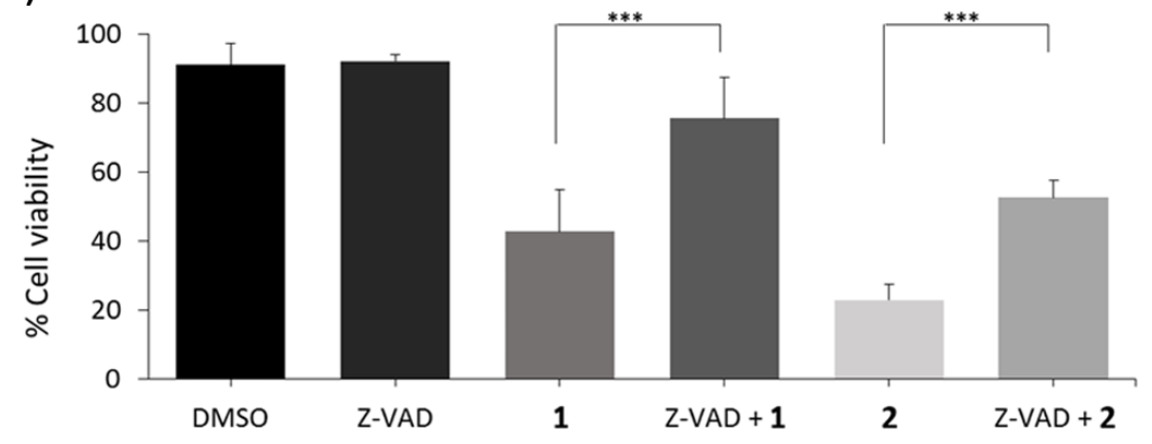




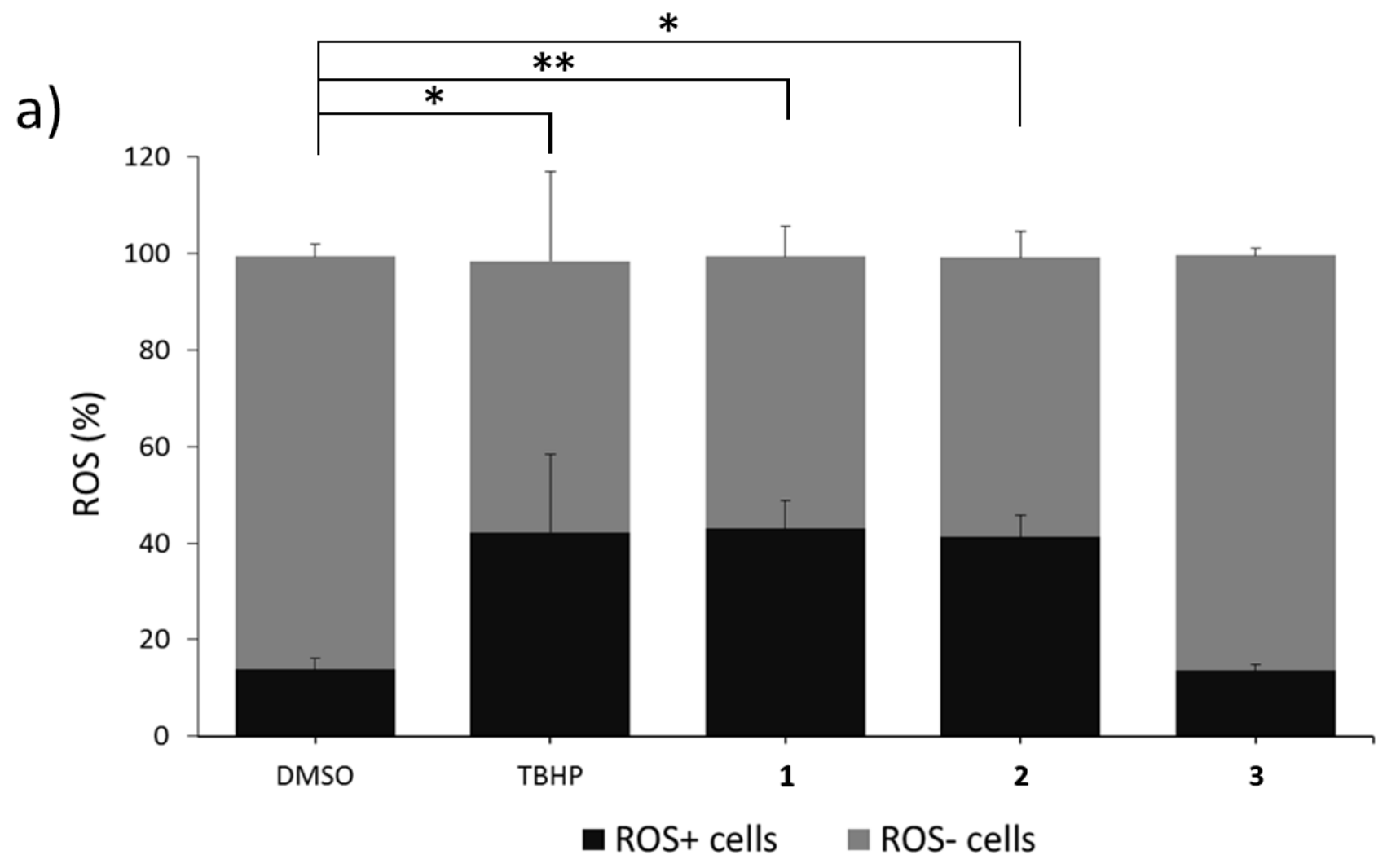

b)

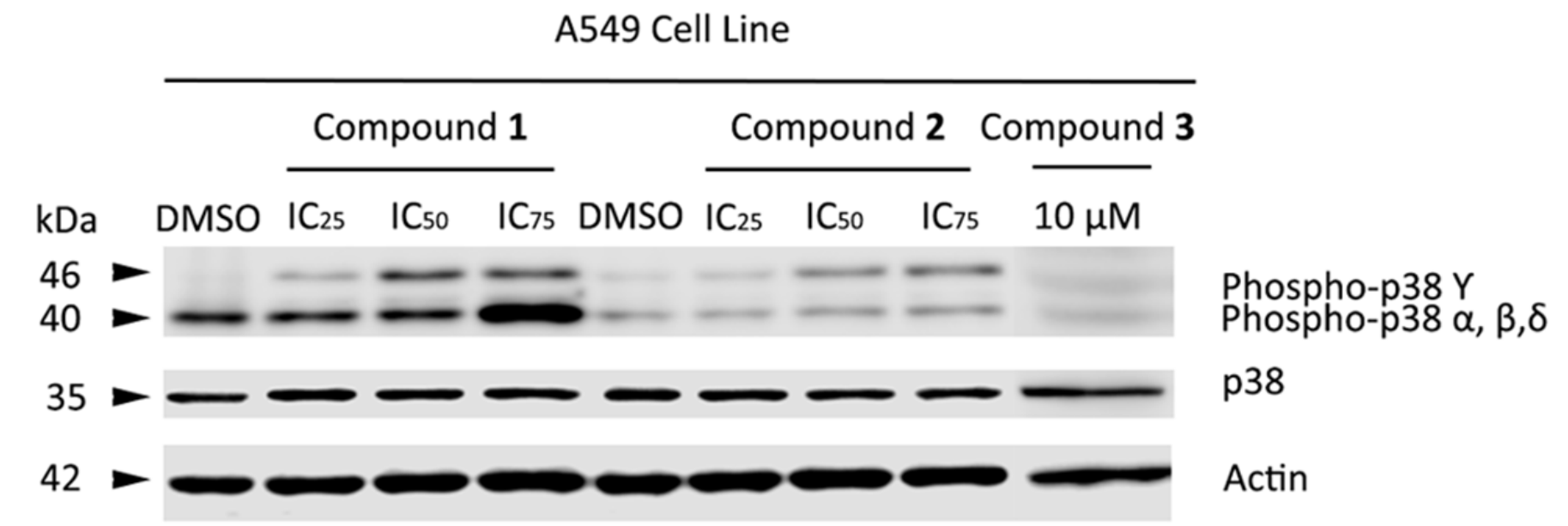

c)

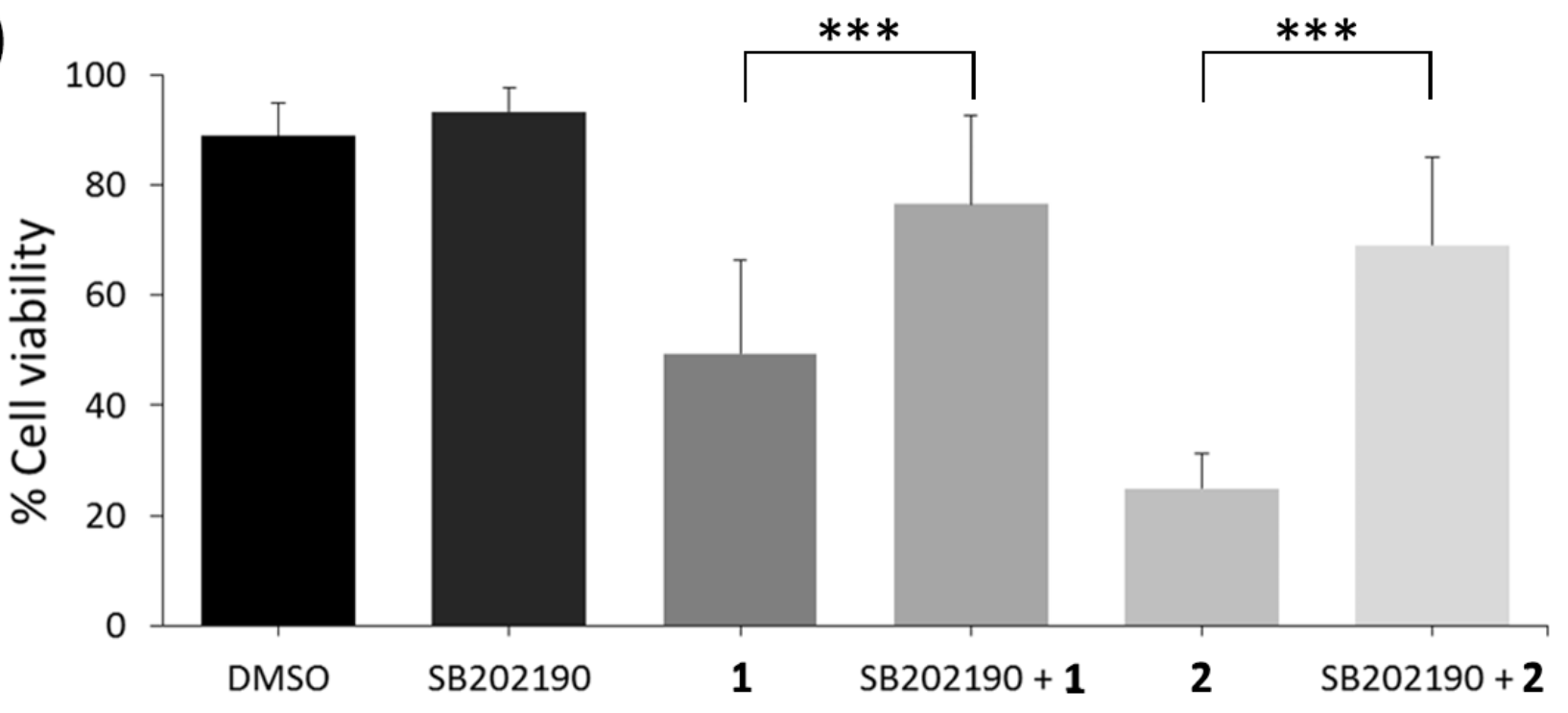



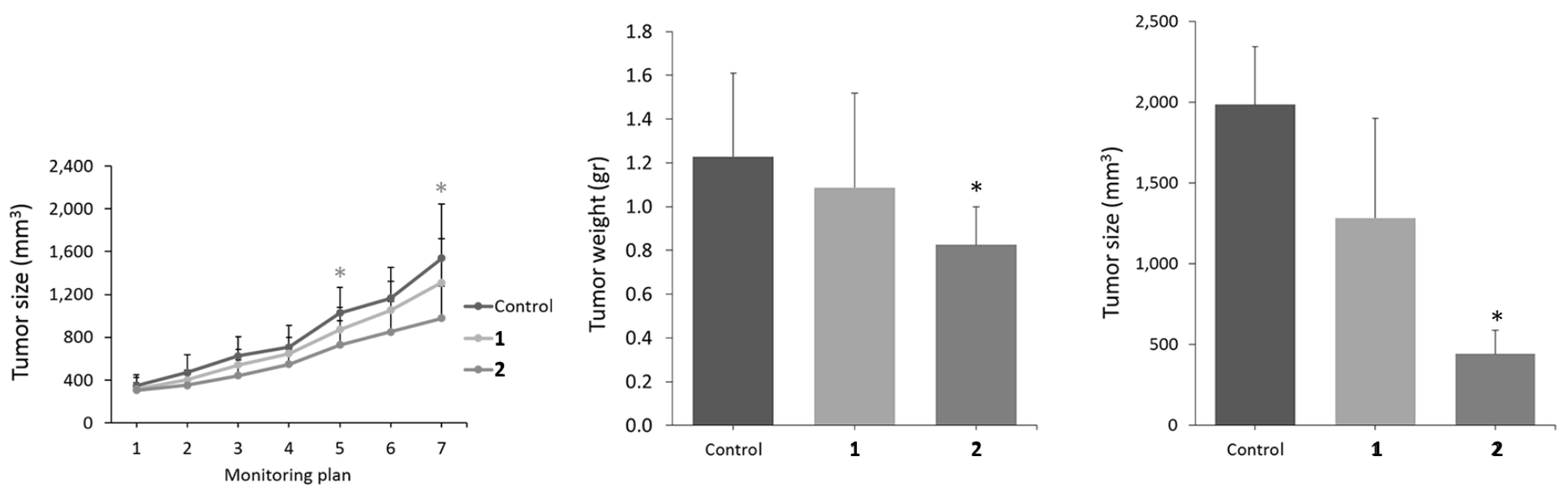

d)

Control

1

2
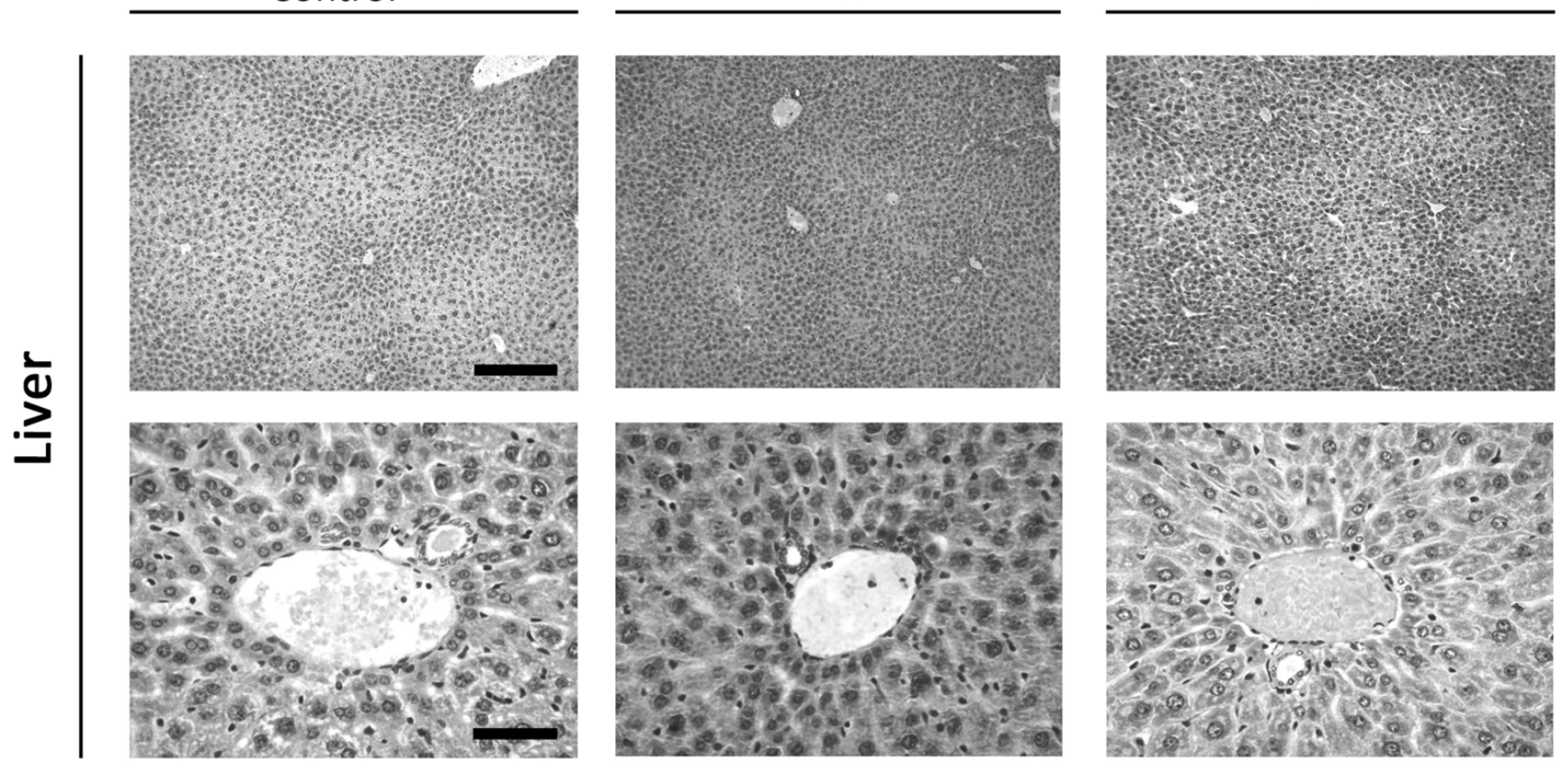

$100 x$
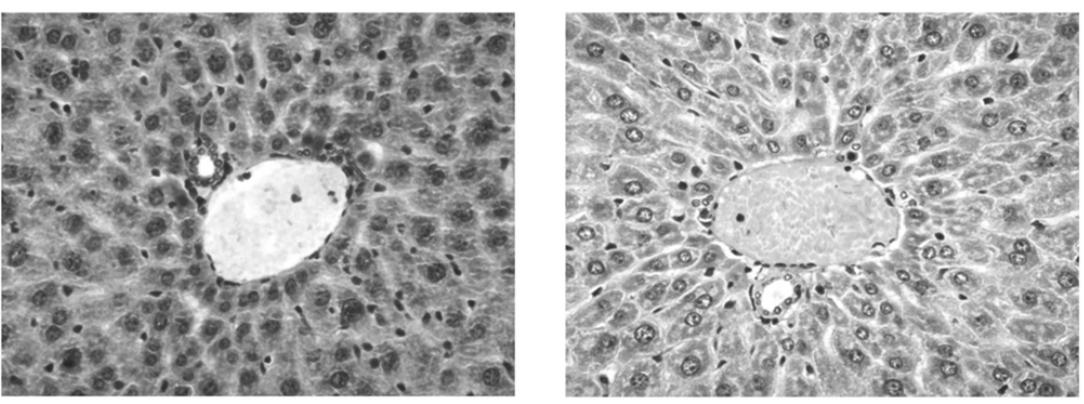

$400 x$
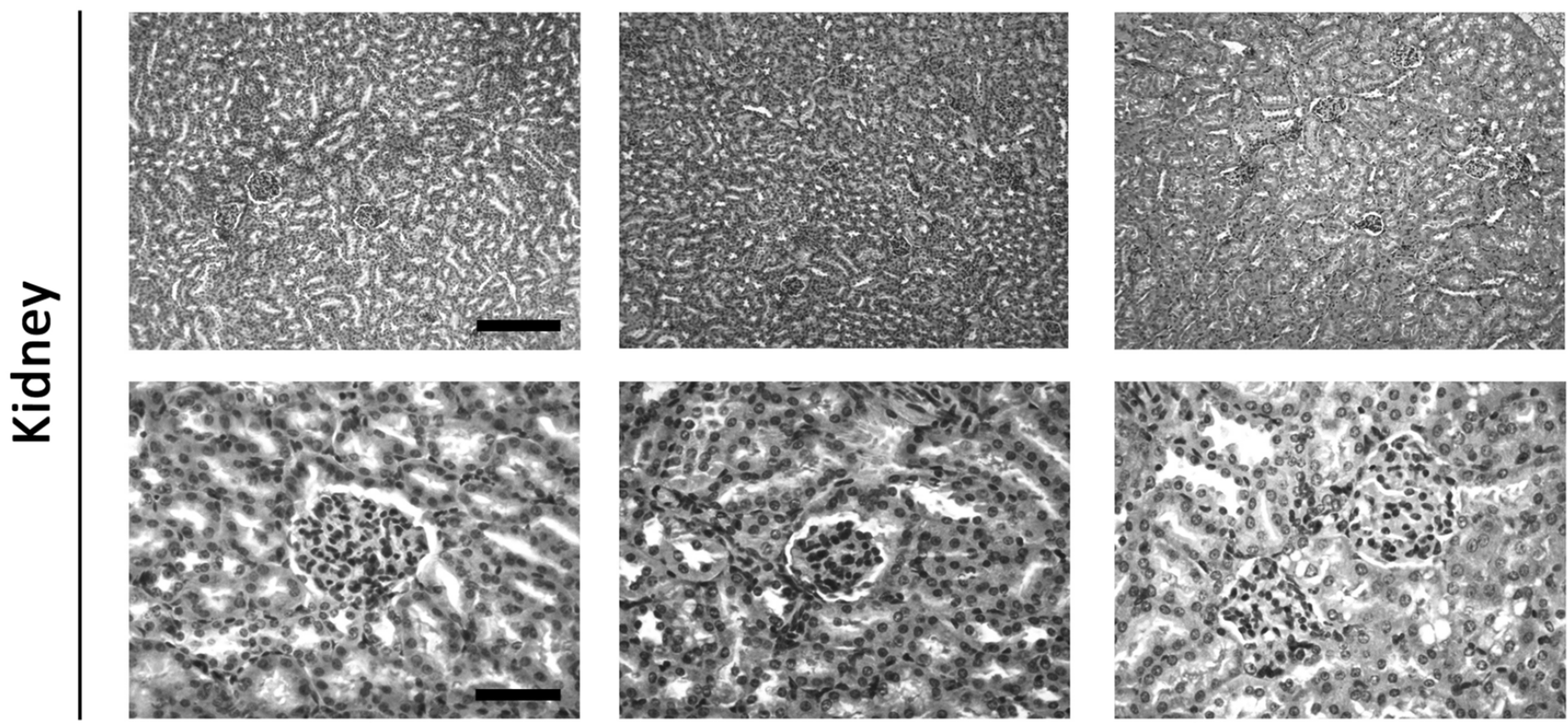

$100 x$
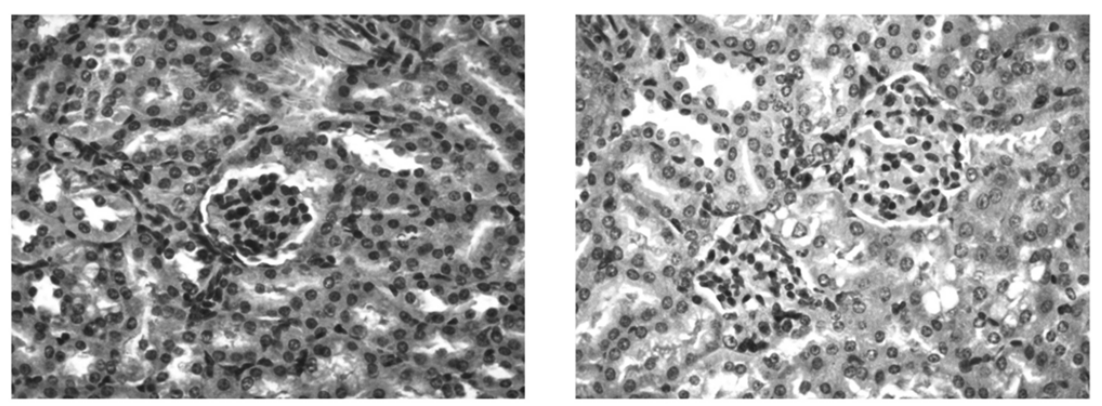